\title{
A first order finite similitude approach to scaled aseismic structures
}

DOI:

10.1016/j.engstruct.2020.111739

\section{Document Version}

Accepted author manuscript

Link to publication record in Manchester Research Explorer

\section{Citation for published version (APA):}

Davey, K., Darvizeh, R., \& Atar, M. (2021). A first order finite similitude approach to scaled aseismic structures. Engineering Structures, 231, [111739]. https://doi.org/10.1016/j.engstruct.2020.111739

\section{Published in:}

Engineering Structures

\section{Citing this paper}

Please note that where the full-text provided on Manchester Research Explorer is the Author Accepted Manuscript or Proof version this may differ from the final Published version. If citing, it is advised that you check and use the publisher's definitive version.

\section{General rights}

Copyright and moral rights for the publications made accessible in the Research Explorer are retained by the authors and/or other copyright owners and it is a condition of accessing publications that users recognise and abide by the legal requirements associated with these rights.

\section{Takedown policy}

If you believe that this document breaches copyright please refer to the University of Manchester's Takedown Procedures [http://man.ac.uk/04Y6Bo] or contact uml.scholarlycommunications@manchester.ac.uk providing relevant details, so we can investigate your claim.

\section{OPEN ACCESS}




\title{
A first order finite similitude approach to scaled aseismic structures
}

\author{
K. Davey, R. Darvizeh, M. Atar \\ School of Mechanical, Aerospace and Civil Engineering, \\ The University of Manchester, ManchesterM13 9PL, UK
}

\begin{abstract}
For many decades the designs of earthquake-resistant (aseismic) structures have been influenced by scaled experiments, underpinned by the theory of dimensional analysis. Although scaled experiments still play an important role, they are recognised to suffer shortcomings, which are particularly severe when scaling ratios are pronounced. The issue is one of scale effects and the inability of dimensional analysis to offer any solution in their presence.

This paper is concerned with a new theory for the analysis of aseismic structures that is founded on the metaphysical concept of space scaling, where beams, substructures or buildings etc. are contracted through the mechanism of space contraction. Although space contraction is evidently practically impossible the theory describes the effects of such a process on the underpinning governing mechanics involved. Unlike dimensional analysis the approach which is termed finite similitude embraces scale effects and accounts for them by linking experiments at more than one scale.

It is demonstrated in this work how it is possible to reconstruct full-scale behaviour by means of two scaled experiments of a selected beam, column and multi-storey structure when subjected to dynamic loading conditions.
\end{abstract}

Keywords: finite similitude, aseismic structures, dimensional analysis, earthquakes, scaled experimentation. 


\section{INTRODUCTION}

For massive structures like sturdy long bridges and skyscrapers, earthquake testing methods such as spot tests or indoor tests have become more challenging and less feasible. In such cases, testing scaled models is more practical, easier to implement and is a cost-effective option and can often be the only solution where testing the real-life prototype is impossible. Scaled experimentation continues to play a significant role in process, product design and testing for components and products but is recognized to suffer from severe limitations. The difficulty with scaling arises mainly from the non-linear relationships between the physical constraints imposed on any scaled physical system. The most readily observable changes that take place are those associated with geometric measures with length changing linearly, area quadratically and volume cubically with scale. This means for example that under a scaled contraction, the body forces will lessen at a much faster rate than the forces on the surface. The complexity of the multitude of changes involved cast a significant shadow over the reliability of scaled experimentation and this issue along with the rise of computational modelling has undoubtedly led to a diminution of scaled physical modelling trials in recent times.

The issue is well appreciated by the academic and industrial communities and the founding bedrock of modern-scaled experimentation is dimensional analysis and the concept of similarity. Similar structures behave identically and through the application of dimensional analysis, similarity can be sought [1]. Rather unfortunately similarity is seldom possible and dimensional analysis provides no solution when any two structures are not similar. In some respects, the theory that underpins scaled experimentation has not changed fundamentally for over a century and dimensional analysis remains the only ubiquitous scaling theory. Dimensional analysis is underpinned by the Buckingham Pi theorem [2] and should the dimensionless quantities (Pi groups) coincide for the full-scale and scaled systems, then the two processes are denoted similar. However, this seldom happens in practice [3] and the approach has to-date had little success in complex structural applications. Although structural engineering has been a field of study for many of hundreds if not over a thousand years, earliest attempts at scaled experimentation prior to the adoption of scaling rules were somewhat rudimentary and highly inaccurate. The work of Buckingham [2] provided the foundation for more realistic scaled models and the first application of the scaled method can again be attributed to him [4], although for a purely theoretically study. A significant increase in the number of publications about scaled methods followed the seminal work of Buckingham [2]. 
A number of scaling approaches [5], [6] have materialised over the years and these include: energy methods (EM), similitude and asymptotic models for structural acoustic research applications (SAMSARA), empirical similarity method (ESM) and sensitivity analysis (SA). Each has their own merits and demerits, but none match the simplicity and ubiquity of dimensional analysis. Moreover, a fundamental concern with all these approaches is that they cannot accommodate to any significant degree scale effects. Scale effects are those changes in behaviour that take place with scale. and recent attempts (e.g. empirical similarity [6], model variation [7], sensitivity analysis [8]) to address the problem of scale effects are to a large extent unsatisfactory being founded on perturbations around the standard definition of similarity [5]. The difficulty associated with scale effects has meant modern-day researchers adopting altogether different methodologies with the application and development of sophisticated computational approaches. Computation however does not altogether bypass experimentation and for those processes involving complex material behaviour, uncertainties, unknown behaviour etc., experimentation still plays a critical role. As noted by Simitses and Rezaeepazhand [9], some systems are so complex to make it almost impossible to make simplifying assumptions for direct simulation. Complex material behaviour has led to the emergence of a plethora of constitutive models and multi-scale approaches [10]-[12]. It is beyond contention that computational approaches have led to significant advances but solving the problem of scale effects should provide alternative complementary approaches.

Full-scale structural experiments have the advantage of being able to replicate to good accuracy the exact environment and realistic conditions of any situation and represent a very direct approach for the analysis of physical phenomena. Thus, despite the complexities of the physics involved, experiments at full scale can provide precise and valuable data. However, with every increasing advances taking place in engineering and technology, the requirements on most experiments are becoming more involved with financial and time requirements increasing accordingly. This is particularly acute in the case of damage-test experiments, which for any realistic structure can be expensive to perform as repetition is often required.

In the case of seismic structural testing the transfer of information from the scaled model to the structure is required to follow scaling rules if results are to be meaningful. Scaling however has its own limitations, where several aspects of the structure cannot always be scaled as a result of constraints on testing equipment (e.g. shake table) or unavailability of materials with the required properties etc. In order to understand the behaviour of the structure under earthquake loading, present-day tests are carried out and interpreted with the application of well-researched scaling relationships. There exist many interesting seismic structural studies involving shake tables, the application of scaling rules and dimensional 
analysis. Sharma et al. [13] for example analysed a three-storey scaled model by applying scaling laws and dimensional analysis theory to investigate the behaviour of the model. Similarly, a scaled model was constructed by Guerrero et al. [14], which was limited to 1/10 of the full-size structure due to size limitations and the cost of the experimental equipment. The prototype structure analysed in this reference is located in Mexico City as stated by Guerrero et al. [15]. Similarly, shake-table testing and studies have been carried out on a one-storey, single-bay steel frame by Nader and Astaneh-Asl [16]. They analysed the performance of steel structures under varying earthquake loadings simulated at smaller scales experimentally. In reference [10] an investigation into the effects of various material and section-level parameters on the structural response metrics was achieved by utilising the financial and logistical benefits offered by small-scale testing (1/8-scale factor). Other studies involving shake table testing are those done on: one-bay, two-storey steel frames, investigating such things as second-order inelastic behaviours [17]; scaling guidelines for modern unreinforced masonry buildings with hollow clay brick units (1/2 scale factor) [18] and; testing of a 1/50 scaled model under one and two dimensional base excitations and gradually increased amplitudes [19]. The main difficulty with all these studies is that they are limited by the inability of dimensional analysis to account for scale effects and the ad hoc nature of the scaling rules needed to extend the analysis.

This paper is concerned with a completely new concept for scaled experimentation termed finite similitude [20]-[25] and attempts to link experiments at more than one scale in a systematic manner. The work here builds on earlier work on scaling (now termed zerothorder finite similitude) that has been applied in the areas of impact mechanics [24], powder compaction [20], biomechanics [23] and metal forming [22], and involves only one scaled experiment. The work presented here is the first application of finite similitude to seismic studies with a more advanced version of the finite-similitude theory involving two scaled experiments.

The finite similitude theory is founded on the metaphysical concept of space scaling which is introduced in Section 2. The focus on space leads to an analysis method that is innately tied to the effects of space contraction, i.e. it naturally leads to control volume concepts in Section 2.2. At first sight the focus on control volumes and transport equations might appear somewhat removed from structural analysis but it is simply a consequence of the path the theory takes to move from space, to a moving region of space (control volume) to transport equations (laws of nature) to field identities and ultimately to structural analysis. The most critical step in the finite-similitude approach as discussed in Section 2.3 for structural mechanics is the projection of the trial-space (where the scaled experiment resides) physics 
onto the physical space (where the full-scale structure resides). This projection reveals in one form or another, scale dependences, explicitly for geometric measures such as area and volume but implicitly for scalar, vector and tensor fields such as density, displacement and stress, respectively. The problem of scaling becomes one of finding these dependencies, which is achieved in this paper through a new form of similarity (first-order finite similitude) as described in Section 3. The solving of the new differential similarity identity is shown in Section 3.1, where physically intuitive field relationships are revealed. One of the features of finite similitude is that it does not concern itself with constitutive equations as it reconstructs all fields in the physical space; this along with application practicalities for the theory are presented in Section 3.1 and Appendix A. Sections 4, 5 and 6 examine the behaviours of a beam, column and three-storey structure, respectively when subject to dynamic excitation to illustrate how the theory can replicate full-scale behaviours. Introduced in Section 5.2 and Appendix B is a new proportional-fields assumption for the determination of similitude parameters. The paper ends with a conclusions section.

\section{THE THEORETICAL FOUNDATION OF FINITE SIMILITUDE}

The finite similitude theory is founded on a metaphysical concept that cannot be physically enacted. However, the purpose of metaphysical-space scaling is threefold; firstly it is to provide a physically intuitive approach to scaled experimentation, where it is possible to imagine the structure of interest being contracted or expanded by the contraction or expansion of space. Secondly, it facilitates the precise mathematical description of scaling, where its effect can be precisely relayed to the underpinning physics of a structure. Thirdly, since in principle, space can be metaphysically distorted in different ways, it introduces a high degree of flexibility, that is simply absent from competing methods.

\subsection{The metaphysics of space scaling}

The analysis of any structure begins with the identification of the inertial frame but at least two inertial frames are involved with scaling, one for the physical and another for the trial space. The starting point therefore is the specification of the trial-space coordinate system (denoted by $\boldsymbol{x}_{t s}$ ) along with a physical-space coordinate system (denoted by $\boldsymbol{x}_{p s}$ ), where the subscripts "ts" and "ps" refer to trial and physical space, respectively. It is assumed here that the coordinate frames, linked to these systems, are orthonormal. It should be appreciated however that structures in the scaled space are viewed from the viewpoint of an external observer, i.e. one unaffected by the scaling process and the choice of coordinate frame is therefore essentially a matter of choice. The overall concept of space scaling is presented in 
Fig. 1, where the orthonormal coordinate frames for the physical space $\left\{\underline{G}_{i}\right\}$ and the trial space $\left\{\underline{g}_{i}\right\}$ are depicted. Shown in the figure are two measures of time $t_{p s}$ and $t_{t s}$ for the physical and trial spaces, respectively. Since Newtonian mechanics is the focus here the existence of absolute time is assumed and it is assumed further that $t_{p s}$ and $t_{t s}$ are related, i.e. a function relationship $t_{p s} \mapsto t_{t s}$ exists, which in differential terms is of the form $d t_{t s}=g d t_{p s}$, where $g$ is a positive parameter. As indicated in the figure, metaphysical scaling is quantified mathematical by a temporally invariant affine map of the form $\boldsymbol{x}_{p s} \mapsto \boldsymbol{x}_{t s}$, which can be represented in differential terms by $d \boldsymbol{x}_{t s}=F \cdot d \boldsymbol{x}_{p s}$, where in suffix notation this is $d x_{t s}^{i}=F_{j}^{i} d x_{p s}^{j}$. Here the matrix $F$ is both spatially and temporally invariant to reflect the focus on scaled experimentation.

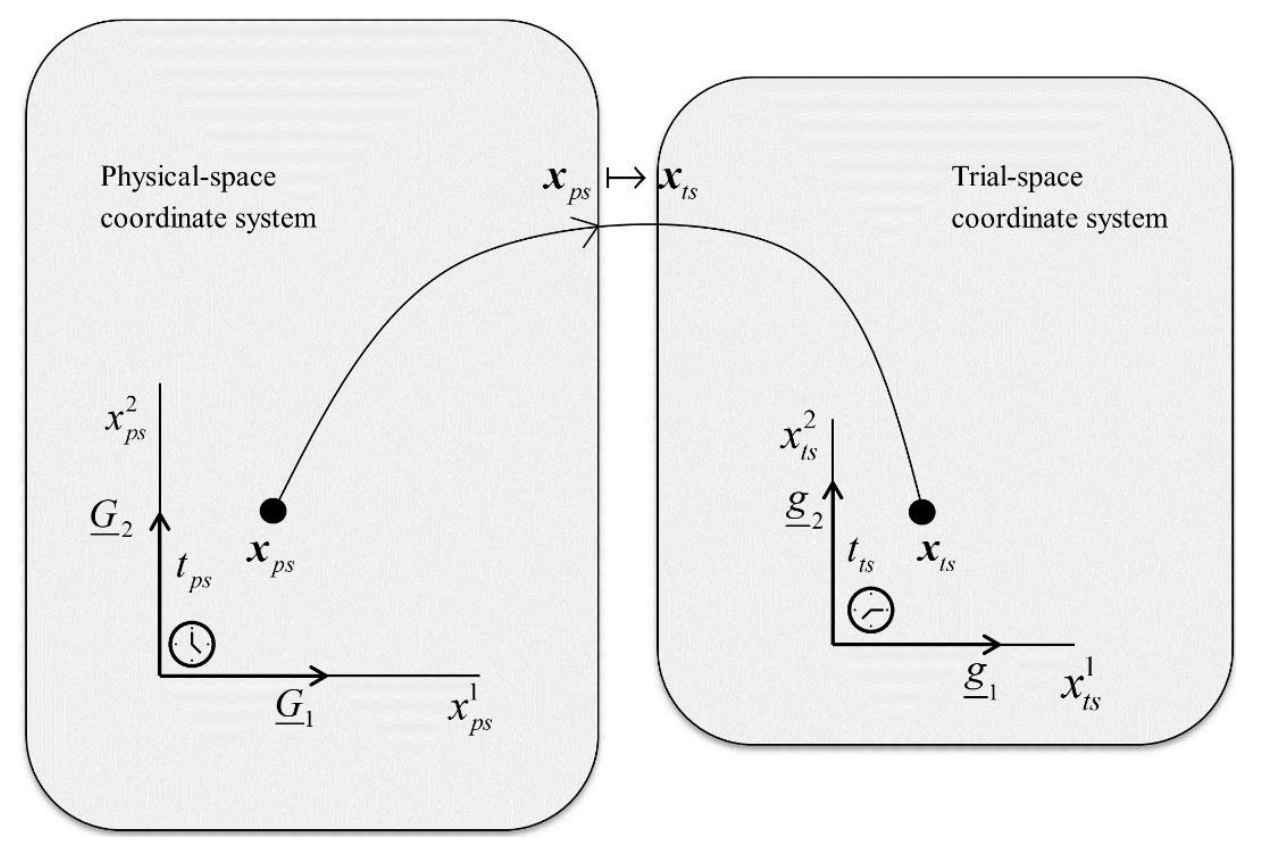

Figure 1. Metaphysical space-scaling concept and inertial coordinate systems

The nature of the space scaling is dictated by the matrix $F$ and anisotropic scaling is indeed possible where geometric similarity as it is traditionally known can be broken. The focus here however is on isotropic scaling with $F=\beta I$, where $I$ is a unit matrix and $\beta$ is a positive parameter that dictates the extent of linear scaling taking place. The effect of $\beta$ on the physical space is illustrated in Fig. 2, with $0<\beta<1$ indicating contraction, $\beta=1$ for no scaling and $1<\beta$ for expansion. 


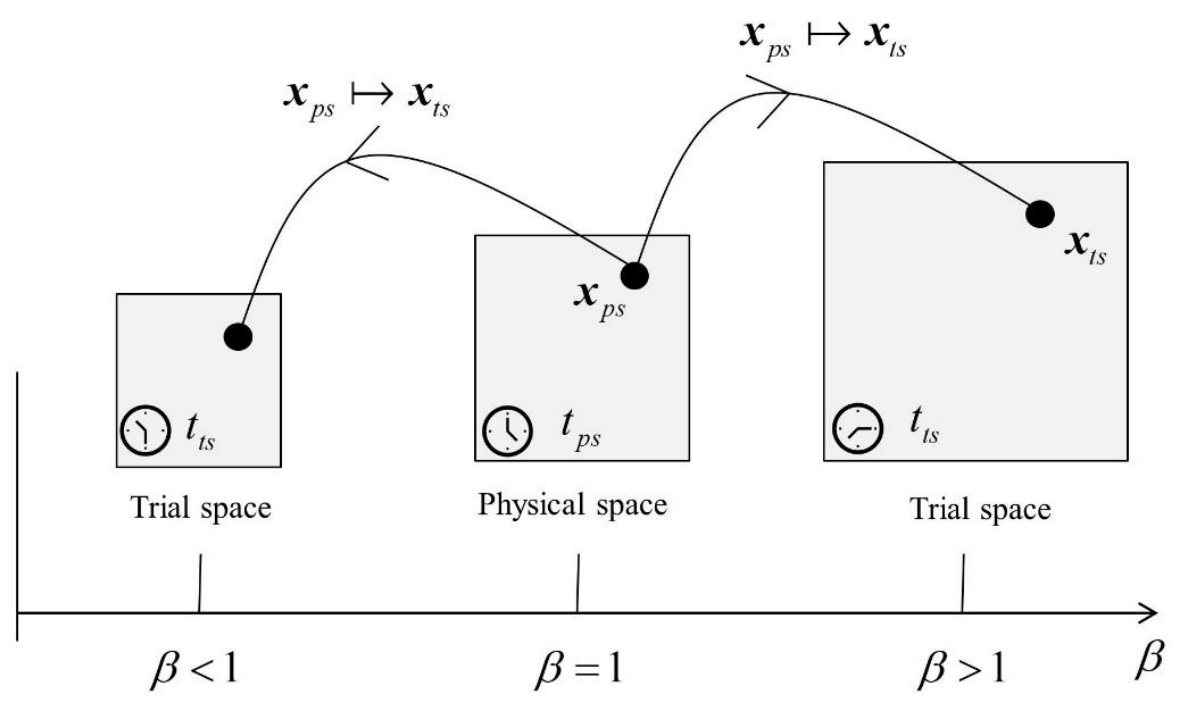

Figure 2 . The role played by $\beta$ in space scaling.

With the establishment and quantification of space scaling it is now possibly to relate the differential-geometric measures of volume and area, i.e. $d V_{t s}=\beta^{3} d V_{p s}$ and $\boldsymbol{n}_{t s} d \Gamma_{t s}=\beta^{2} \boldsymbol{n}_{p s} d \Gamma_{p s}$, where, $d V_{t s} d \Gamma_{t s}$ and $\boldsymbol{n}_{t s}$ refer to volume and area measures, and a unit normal vector, respectively in the trial space; subscript "ps" identifies like quantities in the physical space.

\subsection{The mathematics of control volume motion}

To relate analyses of structures in the trial and physical spaces it is first necessary to be able to identify connections between two regions of space. This can be achieved by means of control volumes, which are regions of space that can move and distort. Control volumes invariably lead to transport equations which at first sight may appear somewhat remote from traditional structural analysis, which is the focus of study here. However, transport equations provide the correct description of the underlying laws of nature as they incorporate directly the changes in geometric measures. The motion of a trial-space control volume $\Omega_{t s}^{*}$ can be described mathematically using a velocity field $v_{t s}^{*}$ and by contrasting its location with to a reference control volume $\Omega_{t s}^{* r e f}$. The basic idea is depicted in Fig. 3 and also shown is the $\operatorname{map} \chi_{t s} \mapsto \boldsymbol{x}_{t s}^{*}$ with $\chi_{t s} \in \Omega_{t s}^{*}$ ref and $\boldsymbol{x}_{t s}^{*} \in \Omega_{t s}^{*}$. 


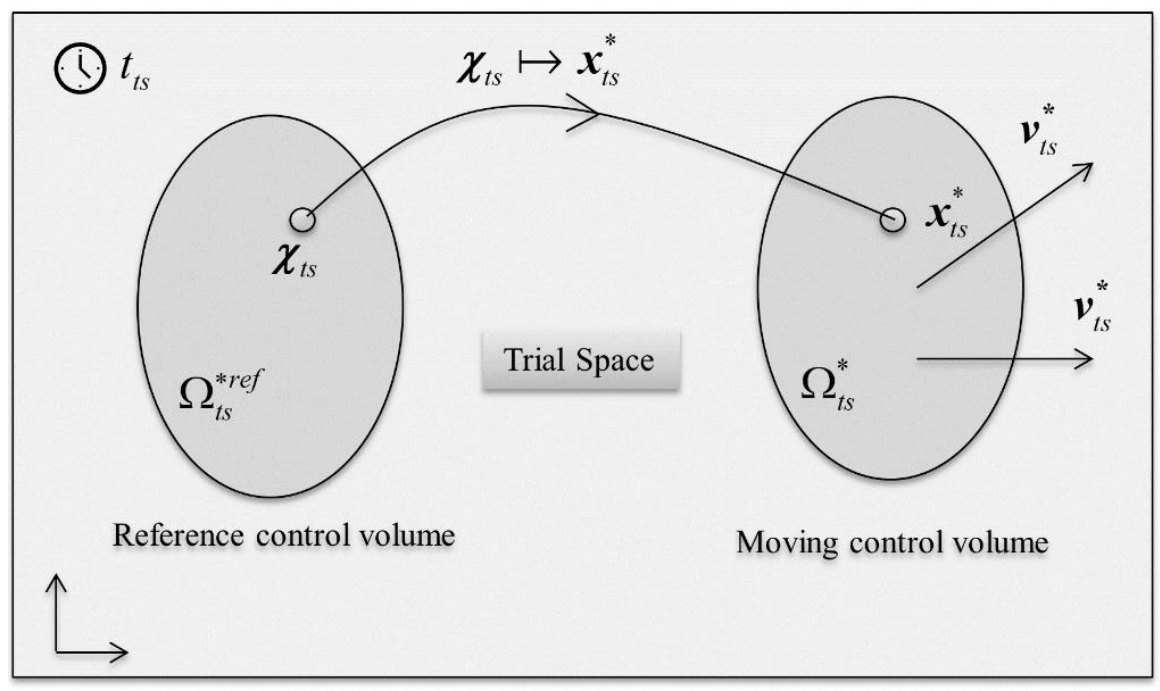

Figure 3. The kinematics of a moving control volume $\Omega_{t s}^{*}$.

The idea presented here is very similar to that employed to describe the motion of a body of mass but in this case, mass is not involved and the body is a moving region of space. The coordinate point $\boldsymbol{x}_{t s}^{*}$ is attached to $\Omega_{t s}^{*}$ and its velocity $\boldsymbol{v}_{t s}^{*}$ is defined by the partial derivative $\boldsymbol{v}_{t s}^{*}=\frac{D^{*} \boldsymbol{x}_{t s}^{*}}{D^{*} t_{t s}}=\left.\frac{\partial \boldsymbol{x}_{t s}^{*}}{\partial t_{t s}}\right|_{\boldsymbol{\chi}_{t s}}$

where the derivative $\frac{D^{*}}{D^{*} t_{t s}}$ is used here to signify a temporal derivative with the reference coordinate $\chi_{t s}$ held constant.

Since a control volume is nothing more than a region of space it is affected in precisely the same manner as the accompanying scaled space and the identity $d \boldsymbol{x}_{t s}^{*}=\beta d \boldsymbol{x}_{p s}^{*}$ is assumed to apply. Moreover, in view of the temporal relationship $d t_{t s}=g d t_{p s}$ it follows that controlvolume velocity fields are related by

$v_{t s}^{*}=g^{-1} \beta v_{p s}^{*}$

which provides synchronous motion of the control volumes $\Omega_{t s}^{*}$ and $\Omega_{p s}^{*}$.

The overall picture displaying the connectivity between reference and moving control volumes in the two spaces along with the synchronous motion is depicted in Fig. 4. Note that the identity $d \boldsymbol{x}_{t s}^{*}=\beta d \boldsymbol{x}_{p s}^{*}$ can be contrasted again the space-scaling identity $d \boldsymbol{x}_{t s}=\beta d \boldsymbol{x}_{p s}$ where it is appreciated that the former unlike the latter is relating moving points. 


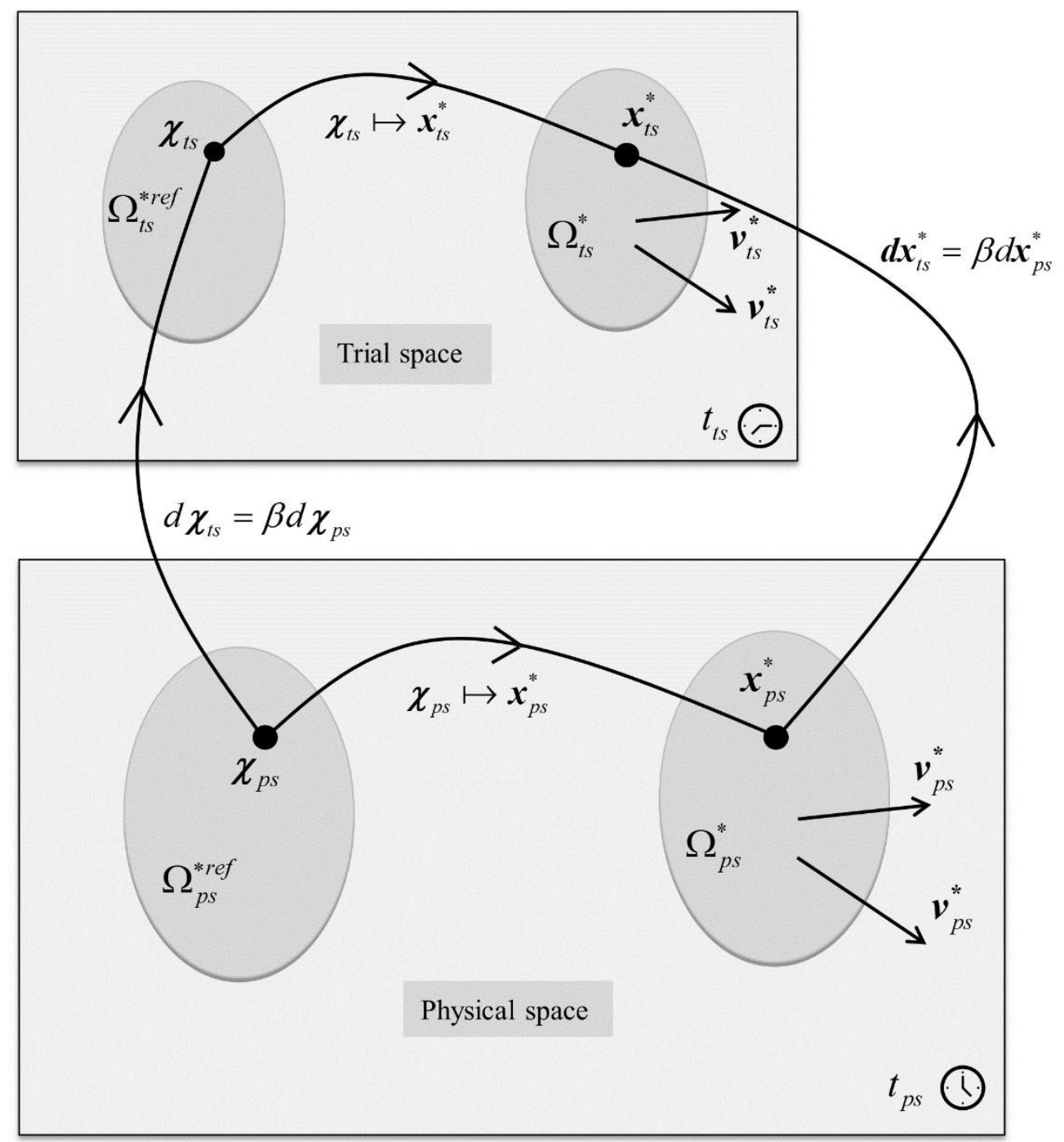

Figure 4. Synchronous motion of control volumes $\Omega_{t s}^{*}$ and $\Omega_{p s}^{*}$.

Eq. (2) is closely related to the law-of-volume identity

$$
\frac{D^{*}}{D^{*} t_{t s}} \int_{\Omega_{t s}^{*}} d V_{t s}^{*}-\int_{\Gamma_{t s}^{*}} \boldsymbol{v}_{t s}^{*} \cdot \boldsymbol{n}_{t s} d \Gamma_{t s}^{*}=0
$$

which is an equation not considered in structural mechanics as it has no field associated with it but nevertheless plays a significant role in finite similitude theory.

It is relatively straightforward to prove that this equation is proportional to the physical space equation

$\frac{D^{*}}{D^{*} t_{p s}} \int_{\Omega_{p s}^{*}} d V_{p s}^{*}-\int_{\Gamma_{p s}^{*}} \boldsymbol{v}_{p s}^{*} \cdot \boldsymbol{n}_{p s} d \Gamma_{p s}^{*}=0$

on substitution of $d V_{t s}^{*}=\beta^{3} d V_{p s}^{*}$ and $\boldsymbol{n}_{t s} d \Gamma_{t s}^{*}=\beta^{2} \boldsymbol{n}_{p s} d \Gamma_{p s}^{*}$ (which follows from $d \boldsymbol{x}_{t s}^{*}=\beta d \boldsymbol{x}_{p s}^{*}$ ) and $d t_{t s}=g d t_{p s}$ along with Eq. (2) to give 
$\frac{D^{*}}{D^{*} t_{t s}} \int_{\Omega_{t s}^{*}} d V_{t s}^{*}-\int_{\Gamma_{t s}^{*}} v_{t s}^{*} \cdot \boldsymbol{n}_{t s} d \Gamma_{t s}^{*}=\frac{\beta^{3}}{g}\left(\frac{D^{*}}{D^{*} t_{p s}} \int_{\Omega_{p s}^{*}} d V_{p s}^{*}-\int_{\Gamma_{p s}^{*}} \boldsymbol{v}_{p s}^{*} \cdot \boldsymbol{n}_{p s} d \Gamma_{p s}^{*}\right)=0$

which evidently confirms proportionality.

The whole idea of the metaphysical space-scaling approach is encapsulated by Eq. (5), where the solution of an equation in one space confirms the solution of the corresponding equation in another.

\subsection{Scaled structural mechanics in transport form}

The transport equations important to structural mechanics for finite similitude are those concerned with continuity, momentum and movement and take the form

$$
\begin{aligned}
& \frac{D^{*}}{D^{*} t_{t s}} \int_{\Omega_{t s}^{*}} \rho_{t s} d V_{t s}^{*}+\int_{\Gamma_{t s}^{*}} \rho_{t s}\left(\boldsymbol{v}_{t s}-\boldsymbol{v}_{t s}^{*}\right) \cdot \boldsymbol{n}_{t s} d \Gamma_{t s}^{*}=0 \\
& \frac{D^{*}}{D^{*} t_{t s}} \int_{\Omega_{t s}^{*}} \rho_{t s} \boldsymbol{v}_{t s} d V_{t s}^{*}+\int_{\Gamma_{t s}^{*}} \rho_{t s} \boldsymbol{v}_{t s}\left(\boldsymbol{v}_{t s}-\boldsymbol{v}_{t s}^{*}\right) \cdot \boldsymbol{n}_{t s} d \Gamma_{t s}^{*}-\int_{\Gamma_{t s}^{*}} \boldsymbol{\sigma}_{t s} \cdot \boldsymbol{n}_{t s} d \Gamma_{t s}^{*}-\int_{\Omega_{t s}^{*}} \rho_{t s} \boldsymbol{b}_{t s} d V_{t s}^{*}=0 \\
& \frac{D^{*}}{D^{*} t_{t s}} \int_{\Omega_{t s}^{*}} \rho_{t s} \boldsymbol{u}_{t s} d V_{t s}^{*}+\int_{\Gamma_{t s}^{*}} \rho_{t s} \boldsymbol{u}_{t s}\left(\boldsymbol{v}_{t s}-\boldsymbol{v}_{t s}^{*}\right) \cdot \boldsymbol{n}_{t s} d \Gamma_{t s}^{*}-\int_{\Omega_{t s}^{*}} \rho_{t s} \boldsymbol{v}_{t s} d V_{t s}^{*}=0
\end{aligned}
$$

where $\rho_{t s}$ is mass density, $\boldsymbol{v}_{t s}$ is material velocity, $\boldsymbol{u}_{t s}$ is material displacement, $\boldsymbol{\sigma}_{t s}$ is Cauchy stress and $\boldsymbol{b}_{t s}$ is specific-body force (i.e. force per unit mass).

It is usual not to involve the continuity equation in structural mechanics as density is often assumed fixed but in the context of scaling and physical modelling, materials can be substituted and it is necessary to understand what substitutions are allowable hence the reason for its inclusion here. Likewise a separate equation for movement is generally not a feature and this was introduced in reference [26] to bring displacement into the family of transport equations and it is particularly convenient to include this here as displacement is central to describing structural deformation.

The next step and most critically important along the path to scaling identities is the projection of Eqs. (6) onto the physical space, as it is through this mathematical operation that scale dependencies are exposed. Consider then the substitution of $d V_{t s}^{*}=\beta^{3} d V_{p s}^{*}$, $\boldsymbol{n}_{t s} d \Gamma_{t s}^{*}=\beta^{2} \boldsymbol{n}_{p s} d \Gamma_{p s}^{*}, d t_{t s}=g d t_{p s}$ and Eq. (2) into Eq. (6) (and Eq. (3) for completeness) and multiplication throughout by $g$. In addition, Eq. (3) and each of Eqs. (6) are multiplied throughout by non-zero scaling parameters $\alpha_{0}^{1}, \alpha_{0}^{\rho}, \alpha_{0}^{v}$ and $\alpha_{0}^{u}$, respectively; the role of which will be made clear. These operations provide the following four equations: 


$$
\begin{aligned}
& \alpha_{0}^{1} T_{0}^{1}(\beta)=\frac{D^{*}}{D^{*} t_{p s}} \int_{\Omega_{p s}^{*}} \alpha_{0}^{1} \beta^{3} d V_{p s}^{*}-\int_{\Gamma_{p s}^{*}} \alpha_{0}^{1} \beta^{3} \boldsymbol{v}_{p s}^{*} \cdot \boldsymbol{n}_{p s} d \Gamma_{p s}^{*}=0 \\
& \alpha_{0}^{\rho} T_{0}^{\rho}(\beta)=\frac{D^{*}}{D^{*} t_{p s}} \int_{\Omega_{p s}^{*}} \alpha_{0}^{\rho} \beta^{3} \rho_{t s} d V_{p s}^{*}+\int_{\Gamma_{p s}^{*}} \alpha_{0}^{\rho} \beta^{3} \rho_{t s}\left(\boldsymbol{V}_{p s}-\boldsymbol{v}_{p s}^{*}\right) \cdot \boldsymbol{n}_{p s} d \Gamma_{p s}^{*}=0 \\
& \alpha_{0}^{v} T_{0}^{v}(\beta)=\frac{D^{*}}{D^{*} t_{p s}} \int_{\Omega_{t s}^{*}}\left(\alpha_{0}^{v} g^{-1} \beta\right) \beta^{3} \rho_{t s} \boldsymbol{V}_{p s} d V_{t s}^{*}+\int_{\Gamma_{p s}^{*}}\left(\alpha_{0}^{v} g^{-1} \beta\right) \beta^{3} \rho_{t s} \boldsymbol{V}_{p s}\left(\boldsymbol{V}_{t s}-\boldsymbol{v}_{p s}^{*}\right) \cdot \boldsymbol{n}_{p s} d \Gamma_{p s}^{*} \\
& \quad-\int_{\Gamma_{p s}^{*}} \alpha_{0}^{v} g \beta^{2} \boldsymbol{\sigma}_{t s} \cdot \boldsymbol{n}_{p s} d \Gamma_{p s}^{*}-\int_{\Omega_{p s}^{*}} \alpha_{0}^{v} g \beta^{3} \rho_{t s} \boldsymbol{b}_{t s} d V_{p s}^{*}=0 \\
& \alpha_{0}^{u} T_{0}^{u}(\beta)=\frac{D^{*}}{D^{*} t_{p s}} \int_{\Omega_{p s}^{*}}\left(\alpha_{0}^{u} \beta\right) \beta^{3} \rho_{t s} \boldsymbol{U}_{t s} d V_{p s}^{*}+\int_{\Gamma_{t s}^{*}}\left(\alpha_{0}^{u} \beta\right) \beta^{3} \rho_{t s} \boldsymbol{U}_{p s}\left(\boldsymbol{V}_{p s}-\boldsymbol{v}_{p s}^{*}\right) \cdot \boldsymbol{n}_{p s} d \Gamma_{p s}^{*} \\
& -\int_{\Omega_{p s}^{*}}\left(\alpha_{0}^{u} \beta\right) \beta^{3} \rho_{t s} \boldsymbol{V}_{p s} d V_{p s}^{*}=0
\end{aligned}
$$

where $\boldsymbol{V}_{p s}=\beta^{-1} g \boldsymbol{v}_{t s}$ and $\boldsymbol{U}_{p s}=\beta^{-1} \boldsymbol{u}_{t s}$.

The importance of Eqs. (7) should not be understated as they capture all scale dependencies that are a feature of scaled structural mechanics. The appearance of $\beta^{3}$ and $\beta^{2}$ are explicit and are recognised to arise from the change in geometric measures of volume and area. Other dependencies are hidden, which include the fields $\boldsymbol{V}_{p s}(\beta)$ and $\boldsymbol{U}_{p s}(\beta)$ along with other fields. In this context scaling has been transformed into a problem of revealing the behaviour of hidden fields and unlike dimensional analysis embraces the changes that take place in scaled experimentation. The process for revealing hidden dependencies can take one of two routes, with one requiring additional information (i.e. scale effects, boundary condition etc.) about the specific problem under consideration and the other effectively assuming how things behaviour in a global sense on application of a scale invariance. This latter approach is the focus here and is particularly well suited to physical modelling where the idea is to design physical-trial experiments to satisfy the scale invariance imposed.

\section{THE MATHEMATICS OF SCALE INVARIANCES}

The transport Eqs. (7) are of the general form $\alpha_{0}^{\psi} T_{0}^{\psi}=0$, with $\psi$ set to 1, $\rho, v$ and $u$. An obvious $\beta$-invariance for scaling is that $\alpha_{0}^{\psi} T_{0}^{\psi}(\beta)$ does not depend on $\beta$, which in mathematical terms is

$$
\frac{d}{d \beta}\left(\alpha_{0}^{\psi} T_{0}^{\psi}\right) \equiv 0
$$


where the equality sign " $\equiv "$ signifies that the derivative is identically zero.

Zeroth-order finite similitude refers to a system of transport equations that satisfies this particular identity and details on its application can be found in reference [20]-[22], [24], [25]. The "initial conditions" for Eq. (8) are taken to be the physical system at $\beta=\beta_{0}=1$. Note for Eqs. (7) that the requirement for $\alpha_{0}^{\psi} T_{0}^{\psi}(1)=0$ to match the physical system imposes the following constraints on the scaling parameters: $\alpha_{0}^{1}(1)=\alpha_{0}^{\rho}(1)=\alpha_{0}^{v}(1)=\alpha_{0}^{u}(1)=1$ along with $g(1)=1$ since $d t_{t s}(1)=g(1) d t_{p s}=d t_{p s}$. Similarly the fields are required to satisfy: $\rho_{t s}(1)=\rho_{p s}, \boldsymbol{V}_{p s}(1)=\boldsymbol{v}_{p s}, \boldsymbol{U}_{p s}(1)=\boldsymbol{u}_{p s}, \boldsymbol{\sigma}_{t s}(1)=\boldsymbol{\sigma}_{p s}$, and so on for other fields. In the presence of scale effects, Eq. (8) will not be satisfied and $\alpha_{0}^{\psi} T_{0}^{\psi}(\beta)$ will therefore depend on $\beta$ but prior to examining this situation it is important to examine how attempting to satisfy this equation impacts on the scaling parameters $\alpha_{0}^{\psi}(\beta)$. Note that integration of Eq. (8) between $\beta_{1}$ and $\beta_{0}=1$ provides $\alpha_{0}^{\psi} T_{0}^{\psi}\left(\beta_{1}\right) \equiv \alpha_{0}^{\psi} T_{0}^{\psi}\left(\beta_{0}\right)=T_{0}^{\psi}(1)$, i.e. the transport equations at any arbitrary scale $\beta_{1}$ match the full-scale system, which of course is very similar to the invariance offered in dimensional analysis where dimensionless-governing equations do not change with scale. Note also that $\beta$ is eliminated from Eq. (7a) on setting $\alpha_{0}^{1}(\beta)=\beta^{-3}$, which satisfies as required $\alpha_{0}^{1}(1)=1$. The condition $\alpha_{0}^{1}(\beta)=\beta^{-3}$ is a necessary and sufficient requirement for the law of volume to be satisfied at any scale. Turning attention now to Eq. (7b) it is apparent that a necessary but not sufficient condition for satisfying Eq. (8) with $\psi=\rho$ is that $\rho_{p s}=\alpha_{0}^{\rho} \beta^{3} \rho_{t s}$. This condition is not sufficient as Eq. (8) additionally requires $\boldsymbol{V}_{p s}=\boldsymbol{v}_{p s}$, which is assumed not to be satisfied here. Examination of Eqs. (7c) and (7d) provide the necessary (but not sufficient) relationships $\alpha_{0}^{v}=g \beta^{-1} \alpha_{0}^{\rho}$ and $\alpha_{0}^{u}=\beta^{-1} \alpha_{0}^{\rho}$. The identities $\rho_{p s}=\alpha_{0}^{\rho} \beta^{3} \rho_{t s}, \alpha_{0}^{v}=g \beta^{-1} \alpha_{0}^{\rho}$ and $\alpha_{0}^{u}=\beta^{-1} \alpha_{0}^{\rho}$ are to be taken forward to the next level of finite similitude termed first-order finite similitude. The observation that the scaling parameters $\alpha_{0}^{\psi}(\beta)$ have the role of attempting to eliminate $\beta$ from $\alpha_{0}^{\psi} T_{0}^{\psi}(\beta)=0$ suggests that a way forward is to consider the scaling of the identity

$T_{1}^{\psi}=\frac{d}{d \beta}\left(\alpha_{0}^{\psi} T_{0}^{\psi}\right)$

with new scaling parameters $\alpha_{1}^{\psi}(\beta)$ (satisfying $\alpha_{1}^{\psi}(1)=1$ ) and consider then

$$
\frac{d}{d \beta}\left(\alpha_{1}^{\psi} T_{1}^{\psi}\right)=\frac{d}{d \beta}\left(\alpha_{1}^{\psi} \frac{d}{d \beta}\left(\alpha_{0}^{\psi} T_{0}^{\psi}\right)\right) \equiv 0
$$


which is the scaled invariance for first-order finite similitude and was first introduced in reference [27] for impact mechanics.

This approach can lead to higher forms with

$T_{2}^{\psi}=\frac{d}{d \beta}\left(\alpha_{1}^{\psi} T_{1}^{\psi}\right)$

etc. but it will become clear that Eq. (10) leads to the requirement of two scaled structural experiments, so is sufficient for our purposes here.

It is important to note that should Eq. (8) be satisfied (i.e. zeroth-order finite similitude), then Eq. (10) is automatically satisfied which is a desirable feature. Also expanding the derivative on the left-hand side of Eq. (10) gives

$$
\frac{d}{d \beta}\left(\alpha_{1}^{\psi} T_{1}^{\psi}\right)=\frac{d \alpha_{1}^{\psi}}{d \beta} T_{1}^{\psi}+\alpha_{1}^{\psi} \frac{d T_{1}^{\psi}}{d \beta} \equiv 0
$$

which is an expansion of the derivatives of $\alpha_{0}^{\psi} T_{0}^{\psi}$, which at any arbitrary $\beta=\beta_{1}$ can represent (by means of osculation) any other linear combination of the derivatives of $\alpha_{0}^{\psi} T_{0}^{\psi}$ (up to the same order), which is sufficient for scaling purposes and illustrates that there is no better alternative to Eq. (10).

The form of Eq. (10) happens to be ideal for integration by means of divided differences as discussed in the following section, which provides added justification for its form.

\subsection{First-order solutions}

Prior to examining the solution to Eq. (10) for transport Eqs. (7) it is judicious to substitute the constraints $\rho_{p s}=\alpha_{0}^{\rho} \beta^{3} \rho_{t s}, \alpha_{0}^{v}=g \beta^{-1} \alpha_{0}^{\rho}$ and $\alpha_{0}^{u}=\beta^{-1} \alpha_{0}^{\rho}$ to provide

$$
\begin{aligned}
& \alpha_{0}^{\rho} T_{0}^{\rho}(\beta)=\frac{D^{*}}{D^{*} t_{p s}} \int_{\Omega_{p s}^{*}} \rho_{p s} d V_{p s}^{*}+\int_{\Gamma_{p s}^{*}} \rho_{p s}\left(\boldsymbol{V}_{p s}-\boldsymbol{v}_{p s}^{*}\right) \cdot \boldsymbol{n}_{p s} d \Gamma_{p s}^{*}=0 \\
& \alpha_{0}^{v} T_{0}^{v}(\beta)=\frac{D^{*}}{D^{*} t_{p s}} \int_{\Omega_{t s}^{*}} \rho_{p s} \boldsymbol{V}_{p s} d V_{t s}^{*}+\int_{\Gamma_{p s}^{*}} \rho_{p s} \boldsymbol{V}_{p s}\left(\boldsymbol{v}_{p s}-\boldsymbol{v}_{p s}^{*}\right) \cdot \boldsymbol{n}_{p s} d \Gamma_{p s}^{*} \\
& -\int_{\Gamma_{p s}^{*}} \boldsymbol{\Sigma}_{t s} \cdot \boldsymbol{n}_{p s} d \Gamma_{p s}^{*}-\int_{\Omega_{p s}^{*}} \boldsymbol{B}_{t s} d V_{p s}^{*}=0 \\
& \alpha_{0}^{u} T_{0}^{u}(\beta)=\frac{D^{*}}{D^{*} t_{p s}} \int_{\Omega_{p s}^{*}} \rho_{p s} \boldsymbol{U}_{t s} d V_{p s}^{*}+\int_{\Gamma_{t s}^{*}} \rho_{p s} \boldsymbol{U}_{p s}\left(\boldsymbol{v}_{p s}-\boldsymbol{v}_{p s}^{*}\right) \cdot \boldsymbol{n}_{p s} d \Gamma_{p s}^{*}-\int_{\Omega_{p s}^{*}} \rho_{p s} \boldsymbol{V}_{p s} d V_{p s}^{*}=0
\end{aligned}
$$

where $\Sigma_{p s}=\alpha_{0}^{v} g \beta^{2} \boldsymbol{\sigma}_{t s}, \boldsymbol{B}_{t s}=\alpha_{0}^{v} g \beta^{3} \rho_{t s} \boldsymbol{b}_{t s}=g^{2} \beta^{-1} \boldsymbol{b}_{t s}$ and where the transfer $\left(\boldsymbol{V}_{p s}-\boldsymbol{v}_{p s}^{*}\right) \cdot \boldsymbol{n}_{p s}$ in the momentum and movement equations is approximated by the zeroth-order expression 
$\left(\boldsymbol{v}_{p s}-\boldsymbol{v}_{p s}^{*}\right) \cdot \boldsymbol{n}_{p s}$ to reflect the fact that the term $\boldsymbol{V}_{p s}\left(\boldsymbol{V}_{p s} \cdot \boldsymbol{n}_{p s}\right)$ is negligible in structural mechanics but also to avoid the necessity to consider higher forms of similitude.

The approach to solving Eq. (10) is to apply divided differences first to Eq. (9) along with a mean-value theorem for integration to provide

$$
\begin{aligned}
& \alpha_{1}^{\psi} \mathbf{T}_{1}^{\psi}\left(\beta_{2}^{1}\right) \equiv \alpha_{1}^{\psi}\left(\beta_{2}^{1}\right) \frac{\alpha_{0}^{\psi} \mathbf{T}_{0}^{\psi}\left(\beta_{1}\right)-\alpha_{0}^{\psi} \mathbf{T}_{0}^{\psi}\left(\beta_{2}\right)}{\beta_{1}-\beta_{2}} \\
& \alpha_{1}^{\psi} \mathbf{T}_{1}^{\psi}\left(\beta_{1}^{0}\right) \equiv \alpha_{1}^{\psi}\left(\beta_{1}^{0}\right) \frac{\alpha_{0}^{\psi} \mathbf{T}_{0}^{\psi}\left(\beta_{0}\right)-\alpha_{0}^{\psi} \mathbf{T}_{0}^{\psi}\left(\beta_{1}\right)}{\beta_{0}-\beta_{1}}
\end{aligned}
$$

where $\beta_{2} \leq \beta_{2}^{1} \leq \beta_{1}$ and $\beta_{1} \leq \beta_{1}^{0} \leq \beta_{0}$ with $\beta_{2}$ and $\beta_{1}$ being scales for trial-space experimentation and $\beta_{0}=1$ being at full scale.

In view of Eq. (10) the next divided difference gives zero or equivalently $\alpha_{1}^{\psi} T_{1}^{\psi}\left(\beta_{1}^{0}\right) \equiv \alpha_{1}^{\psi} T_{1}^{\psi}\left(\beta_{2}^{1}\right)$, which on substitution of Eqs. (14) provides after some manipulation

$\alpha_{0}^{\psi} \mathrm{T}_{0}^{\psi}\left(\beta_{0}\right) \equiv \alpha_{0}^{\psi} \mathrm{T}_{0}^{\psi}\left(\beta_{1}\right)+R_{1}^{\psi}\left(\alpha_{0}^{\psi} \mathrm{T}_{0}^{\psi}\left(\beta_{1}\right)-\alpha_{0}^{\psi} \mathrm{T}_{0}^{\psi}\left(\beta_{2}\right)\right)$

where

$$
R_{1}^{\psi}=\left(\frac{\alpha_{1}^{\psi}\left(\beta_{2}^{1}\right)}{\alpha_{1}^{\psi}\left(\beta_{1}^{0}\right)}\right)\left(\frac{\beta_{0}-\beta_{1}}{\beta_{1}-\beta_{2}}\right)
$$

with Eq. (15) providing the sought expression for relating trial-space experiments to the fullscale structure, and where $R_{1}^{\psi}$ takes on the form of a parameter due to indeterminacy of $\alpha_{1}^{\psi}$. Application of Eq. (15) to Eqs. (13) provides the following field identities:

$$
\begin{aligned}
& \boldsymbol{v}_{p s}=\boldsymbol{V}_{p s}\left(\beta_{1}\right)+R_{1}^{\rho}\left(\boldsymbol{V}_{p s}\left(\beta_{1}\right)-\boldsymbol{V}_{p s}\left(\beta_{2}\right)\right) \\
& \boldsymbol{v}_{p s}=\boldsymbol{V}_{p s}\left(\beta_{1}\right)+R_{1}^{v}\left(\boldsymbol{V}_{p s}\left(\beta_{1}\right)-\boldsymbol{V}_{p s}\left(\beta_{2}\right)\right) \\
& \boldsymbol{\sigma}_{p s}=\Sigma_{p s}\left(\beta_{1}\right)+R_{1}^{v}\left(\Sigma_{p s}\left(\beta_{1}\right)-\Sigma_{p s}\left(\beta_{2}\right)\right) \\
& \boldsymbol{b}_{p s}=\boldsymbol{B}_{p s}\left(\beta_{1}\right)+R_{1}^{v}\left(\boldsymbol{B}_{p s}\left(\beta_{1}\right)-\boldsymbol{B}_{p s}\left(\beta_{2}\right)\right) \\
& \boldsymbol{u}_{p s}=\boldsymbol{U}_{p s}\left(\beta_{1}\right)+R_{1}^{u}\left(\boldsymbol{U}_{p s}\left(\beta_{1}\right)-\boldsymbol{U}_{p s}\left(\beta_{2}\right)\right) \\
& \boldsymbol{v}_{p s}=\boldsymbol{V}_{p s}\left(\beta_{1}\right)+R_{1}^{u}\left(\boldsymbol{V}_{p s}\left(\beta_{1}\right)-\boldsymbol{V}_{p s}\left(\beta_{2}\right)\right)
\end{aligned}
$$


where to arrive at a consistent velocity expression it is required that $R_{1}=R_{1}^{\rho}=R_{1}^{v}=R_{1}^{u}$, which is achieved on setting $\alpha_{1}^{\rho}=\alpha_{1}^{v}=\alpha_{1}^{u}$, and where as mentioned above $\boldsymbol{V}_{p s}=\beta^{-1} g \boldsymbol{v}_{t s}$, $\boldsymbol{U}_{p s}=\beta^{-1} \boldsymbol{u}_{t s}, \boldsymbol{\Sigma}_{p s}=\alpha_{0}^{v} g \beta^{2} \boldsymbol{\sigma}_{t s}$ and $\boldsymbol{B}_{t s}=g^{2} \beta^{-1} \boldsymbol{b}_{t s}$.

The condition $R_{1}^{\rho}=R_{1}^{v}=R_{1}^{u}$ provides a physically-intuitive solution for Eq. (15) in that it indicates that the differences between the experiments as described by transport Eqs. (7) are proportional. The theory provides the fields in Eqs. (17) whose differences are also proportional. The final solution to the scaling problem is rather elegant in its simplicity and all that remains is the details of its application.

\subsection{Consistency and applicability}

One of the features of the transport approach is that it does not depend on nor utilise constitutive laws as it provides all the physical fields needed for the physical space. In small deflection theory for example the identities $d x_{p s}^{i}=\beta_{1}^{-1} d x_{p s}^{i}\left(\beta_{1}\right)=\beta_{2}^{-1} d x_{p s}^{i}\left(\beta_{2}\right)$ and Eq. (17e) provide the strain relationship

$$
\boldsymbol{\varepsilon}_{p s}=\boldsymbol{\varepsilon}_{t s}\left(\beta_{1}\right)+R_{1}\left(\varepsilon_{t s}\left(\beta_{1}\right)-\varepsilon_{t s}\left(\beta_{2}\right)\right)
$$

which confirms that with first-order finite similitude, strains are not required to be identical, which is a feature of dimensional analysis.

Note also that Eq. (17f) and (17e) are consistent since division of the latter by $D t_{p s}=g_{1}^{-1} D t_{p s 1}=g_{2}^{-1} D t_{p s 2}$, with $g_{i}=g\left(\beta_{i}\right)$ and $t_{t s i}=t_{t s}\left(\beta_{i}\right)$ gives

$$
\frac{D \boldsymbol{u}_{p s}}{D t_{p s}}=g_{1} \frac{D \boldsymbol{U}_{p s 1}}{D t_{t s 1}}+R_{1}\left(g_{1} \frac{D \boldsymbol{U}_{p s 1}}{D t_{t s 1}}-g_{1} \frac{D \boldsymbol{U}_{p s}}{D t_{t s 1}}\right)=\boldsymbol{V}_{p s}\left(\beta_{1}\right)+R_{1}\left(\boldsymbol{V}_{p s}\left(\beta_{1}\right)-\boldsymbol{V}_{p s}\left(\beta_{2}\right)\right)
$$

as expected, where $\frac{D}{D t_{t s}}$ and $\frac{D}{D t_{p s}}$ means material derivatives.

Before discussing the practical application of the theory, it is useful at this point to tabulate for both zeroth and first-order theories the important relationships, which are brought together in Table 1. It is worth noting that despite the relative complexity involved in the derivation of the field relationships in Table 1, their application transpires to be relatively straightforward. A detailed set of instructions for the application of both zeroth and first order finite similitude is presented in Appendix A. A particularly nice feature of the proposed similitude approach is that it can be applied directly to theoretical, numerical, and experimental results. The examples presented in the next three sections are purposely chosen to illustrate this point but also to demonstrate the relative ease of applicability for problems of increasing complexity. 
Table 1. Important zeroth and first-order finite similitude identities

\begin{tabular}{|c|c|c|}
\hline \multicolumn{3}{|c|}{ Finite similitude relationships } \\
\hline \multirow[b]{2}{*}{ Density } & $0^{\text {th }}$-order & $\rho_{p s}=\alpha_{01}^{\rho} \rho_{t s 1} \beta_{1}^{3}$ \\
\hline & $1^{\text {st }}$-order* & $\rho_{p s}=\alpha_{01}^{\rho} \rho_{t s 1} \beta_{1}^{3}+R_{1}\left(\alpha_{01}^{\rho} \rho_{t s 1} \beta_{1}^{3}-\alpha_{02}^{\rho} \rho_{t s 2} \beta_{2}^{3}\right)$ \\
\hline \multirow{2}{*}{ Displacement } & $0^{\text {th }}$-order & $\boldsymbol{u}_{p s}=\beta_{1}^{-1} \boldsymbol{u}_{t s 1}$ \\
\hline & $1^{\text {st }}$-order & $\boldsymbol{u}_{p s}=\beta_{1}^{-1} \boldsymbol{u}_{t s 1}+R_{1}\left(\beta_{1}^{-1} \boldsymbol{u}_{t s 1}-\beta_{2}^{-1} \boldsymbol{u}_{t s 2}\right)$ \\
\hline \multirow{2}{*}{ Strain } & $0^{\text {th }}$-order & $\boldsymbol{\varepsilon}_{p s}=\boldsymbol{\varepsilon}_{t s 1}$ \\
\hline & $1^{\text {st }}$-order & $\varepsilon_{p s}=\varepsilon_{t s 1}+R_{1}\left(\varepsilon_{t s 1}-\varepsilon_{t s 2}\right)$ \\
\hline \multirow{2}{*}{ Stress } & $0^{\text {th }}$-order & $\boldsymbol{\sigma}_{p s}=\alpha_{01}^{\rho} g_{1}^{2} \beta_{1} \sigma_{t s 1}$ \\
\hline & $1^{\text {st-order }}$ & $\sigma_{p s}=\alpha_{01}^{\rho} g_{1}^{2} \beta_{1} \sigma_{t s 1}+R_{1}\left(\alpha_{01}^{\rho} g_{1}^{2} \beta_{1} \sigma_{t s 1}-\alpha_{02}^{\rho} g_{2}^{2} \beta_{2} \sigma_{t s 2}\right)$ \\
\hline
\end{tabular}

* Not derived in this work

\section{THE SCALING OF A BEAM: AN ANALYTICAL STUDY}

This section serves to provide an initial test on a problem of some simplicity to illustrate the practical implementation of the zeroth and first-order finite similitude theory by the scaling of a cantilever beam. The cantilever beam is depicted in Fig.5 and the goal here is to ascertain whether it is possible to capture the dynamic response of the beam using either one (zerothorder) or two (first-order) trial-space tests. The behaviour of the cantilever beam in the physical space is assumed to be known in this analysis although in practice this might not be the case. It is assumed here that the free vibration response of the cantilever is described by the well-known Euler-Bernoulli solution [28]:

$$
w(x, t)=\sum_{n=1}^{\infty} a_{n} \cos \left(\omega_{n} t\right) \phi\left(x ; \gamma_{n}, L\right)
$$

where the eigenfunctions $\phi\left(x ; \gamma_{n}, L\right)$ are

$$
\phi\left(x ; \gamma_{n}, L\right)=\left[\cosh \left(\gamma_{n} x\right)-\cos \left(\gamma_{n} x\right)\right]-\left(\frac{\sinh \left(\gamma_{n} L\right)-\sin \left(\gamma_{n} L\right)}{\cosh \left(\gamma_{n} L\right)+\cos \left(\gamma_{n} L\right)}\right)\left[\sinh \left(\gamma_{n} x\right)-\sin \left(\gamma_{n} x\right)\right]
$$




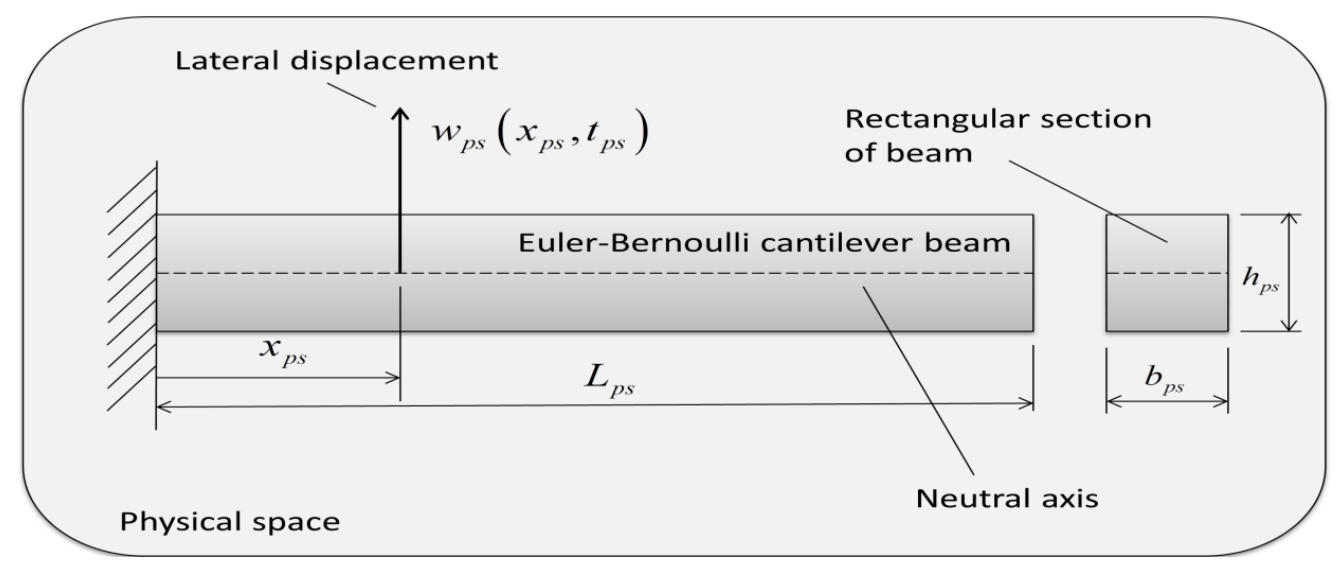

Figure 5. Uniform cantilever beam in the physical space

and where $\gamma_{n} L$ represents the frequency coefficient [28], $\omega_{n}=\left(\gamma_{n} L\right)^{2} \sqrt{E I /\left(\rho A L^{4}\right)}$ are natural frequencies, with Young's modulus $E$, second moment of area $I=\frac{1}{12} b h^{3}$, cross sectional area $A=b h$, mass density $\rho$ and $a_{n}$ are set to capture the initial configuration.

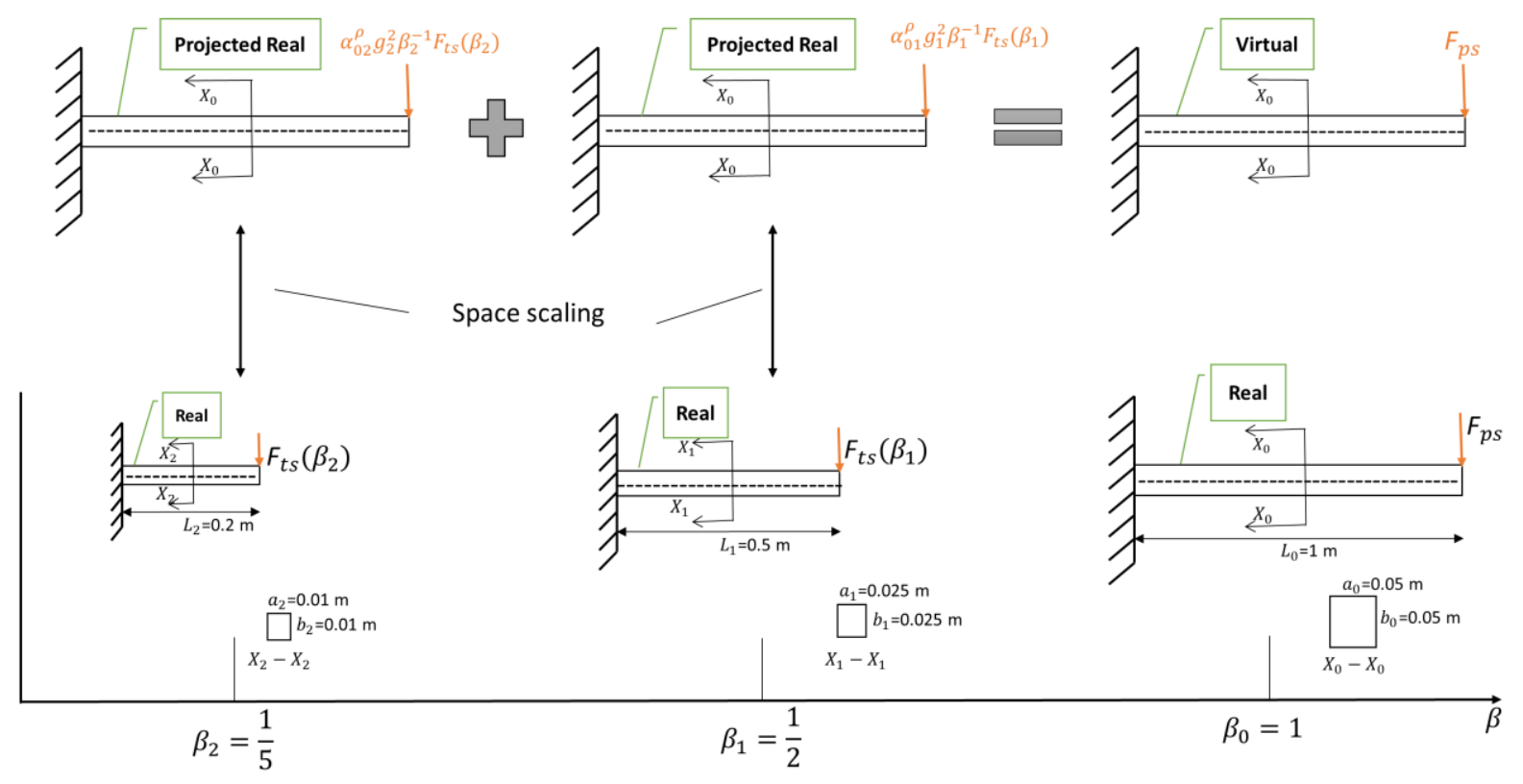

Figure 6. Projected trial and physical space models for the cantilever beam.

An overall view of the scaling process with one or two trial experiments involved is presented in Fig. 6. Shown in the figure is how space scaling is used to project the "real" trial-space experiments into the physical space and their subsequent combination using Eq. (15). Shown also is the effect of space scaling on the load at the free end of the beam with the aim that on combination of the projected beams the force $F_{p s}$ is returned. In the tests that follow three designs are considered at selected scales $\beta_{2}=\frac{1}{5}$ and $\beta_{1}=\frac{1}{2}$, and are labelled Designs I, II and III. Design I is limited zeroth-order finite similitude with the same material used for both physical and trial space. Design II is also for identical materials but applies 
first-order scaling with Design III looking at alternative materials typical to physical modelling.

\subsection{Design I: Zeroth order with identical beam materials}

Applying the zeroth-order procedure presented in Appendix A provides:

(i) The physical space cantilever is made of steel with properties listed at Table 2 and has dimensions $a_{p s}=b_{p s}=0.05 \mathrm{~m}$ and $L_{p s}=1 \mathrm{~m}$ (see Fig. 6.). The initial condition assumed to apply is obtained on setting $a_{n}=$ constant for all $n \geq 1$ with the constant specified so that $w_{p s}\left(L_{p s}, 0\right)=0.1 \mathrm{~m}$ and consequently the transient response is described by

$$
w_{p s}\left(x_{p s}, t_{p s}\right)=0.1 \frac{\sum_{n=1}^{\infty} \cos \left(\left(\omega_{p s}\right)_{n} t_{p s}\right) \phi\left(x_{p s} ;\left(\gamma_{p s}\right)_{n}, L_{p s}\right)}{\sum_{n=1}^{\infty} \phi\left(L_{p s} ;\left(\gamma_{p s}\right)_{n}, L_{p s}\right)}
$$

where the function $\phi$ is given in Eqs. (20).

(ii) The dimensional scaling factor $\beta_{1}=\frac{1}{2}$ and consequently the steel (properties in Table 2) trial-space cantilever beam has dimensions $a_{t s 1}=b_{t s 1}=0.025 \mathrm{~m}$ and $L_{t s 1}=0.50 \mathrm{~m}$ as shown in Fig. 6.

(iii) The density and elastic modulus are chosen to be fixed.

(iv) The density and time scaling factors are determined and equate to $\alpha_{01}^{\rho}=8.0$ and $g_{1}=0.5$ so that the identities $\rho_{p s}=\alpha_{01}^{\rho} \rho_{t s 1} \beta_{1}^{3}$ and $E_{p s}=\alpha_{01}^{\rho} g_{1}^{2} \beta_{1} E_{t s 1}$ are satisfied. (see Table 1),

(v) The initial deflection of the beam at the free end is set to $w_{t s 1}\left(L_{t s 1}, 0\right)=0.05 \mathrm{~m}$ in accordance with the displacement identity in Table 1.

(vi) By testing the trial model, its deflection-time behaviour satisfies

$$
w_{t s 1}\left(x_{t s 1}, t_{t s 1}\right)=0.05 \frac{\sum_{n=1}^{\infty} \cos \left(\left(\omega_{t s 1}\right)_{n} t_{t s 1}\right) \phi\left(x_{t s 1} ;\left(\gamma_{t s 1}\right)_{n}, L_{t s 1}\right)}{\sum_{n=1}^{\infty} \phi\left(L_{t s 1} ;\left(\gamma_{t s 1}\right)_{n}, L_{t s 1}\right)}
$$

(vii) The final procedure is the lifting of the trial model response to predict the physical model (e.g. $\left.w_{p s}=\beta_{1}^{-1} w_{t s 1}\right)$.

The results of this study are presented in Fig. 7 with the deflection at the free end captured as function of time; there is perfect match between the projected trial and physical results. 


\subsection{Design II: First order with identical beam materials}

Applying the zeroth-order procedure presented in Appendix B provides:

(i) The steel cantilever beam (properties in Table 2) in the physical space is again considered as in Section 4.1 with initial deflection satisfying Eq. (22) at time $t_{p s}=0$.

(ii) The dimensional scaling factors for the two trial-spaces are set to be $\beta_{1}=\frac{1}{2}$ and $\beta_{2}=\frac{1}{5}$ making the dimensions of the two steel cantilevers (properties listed in Table 2) to be $a_{t s 1}=b_{t s 1}=0.025 \mathrm{~m}, L_{t s 1}=0.50 \mathrm{~m}, a_{t s 2}=b_{t s 2}=0.01 \mathrm{~m}$ and $L_{t s 2}=0.20 \mathrm{~m}$, as illustrated in Fig. 6.

(iii) The density scaling factors $\alpha_{01}^{\rho}$ and $\alpha_{02}^{\rho}$ are set on the basis of zeroth-order assumptions and equate to $\alpha_{01}^{\rho}=8$ and $\alpha_{02}^{\rho}=125$ so that the relationships $\rho_{p s}=\alpha_{01}^{\rho} \beta_{1}^{3} \rho_{t s 1}$ and $\rho_{p s}=\alpha_{02}^{\rho} \beta_{2}^{3} \rho_{t s 2}$ are satisfied.

(iv) The elastic modulus and the initial (or loading) conditions, which are displacement and the force required to cause this displacement are selected to be fixed.

(v) The temporal and first-order scaling factor $g_{1}, g_{2}$ and $R_{1}$ are found to equate to $g_{1}=0.5, g_{2}=0.2 R_{1}=-0.12$. These are arrived by solving the following three algebraic equations:

$$
\begin{aligned}
& E_{p s}=\alpha_{01}^{\rho} g_{1}^{2} \beta_{1} E_{t s 1}+R_{1}\left(\alpha_{01}^{\rho} g_{1}^{2} \beta_{1} E_{t s 1}-\alpha_{02}^{\rho} g_{2}^{2} \beta_{2} E_{t s 2}\right) \\
& F_{p s}=\alpha_{01}^{\rho} g_{1}^{2} \beta_{1}^{-1} F_{t s 1}+R_{1}\left(\alpha_{01}^{\rho} g_{1}^{2} \beta_{1}^{-1} F_{t s 1}-\alpha_{02}^{\rho} g_{2}^{2} \beta_{2}^{-2} F_{t s 2}\right) \\
& w_{p s}^{\text {end }}=\beta_{1}^{-1} w_{t s 1}^{\text {end }}+R_{1}\left(\beta_{1}^{-1} w_{t s 1}^{\text {end }}-\beta_{2}^{-1} w_{t s 2}^{\text {end }}\right)
\end{aligned}
$$

where the end forces are set to $F_{t s 2}=0.10 F_{p s}, F_{t s 1}=0.20 F_{p s}$ (see Fig. 6) and the initial end displacements $w_{t s 2}^{\text {end }}$ and $w_{t s 1}^{\text {end }}$ are determined on the basis of these forces at static equilibrium.

(vi) The initial conditions for the first and second trial models are set on the basis of end deflections $w_{t s 2}^{\text {end }}$ and $w_{t s 1}^{\text {end }}$, i.e. $w_{t s 2}\left(L_{t s 2}, 0\right)=w_{t s 2}^{\text {end }}$ and $w_{t s 1}\left(L_{t s 1}, 0\right)=w_{t s 1}^{\text {end }}$.

(vii) Transient deflection of the beams in the trial space satisfy similar looking equations to Eq. (22) and explicitly are

$$
w_{t s i}\left(x_{t s i}, t_{t s i}\right)=w_{t s i}^{\text {end }} \frac{\sum_{n=1}^{\infty} \cos \left(\left(\omega_{t s i}\right)_{n} t_{t s i}\right) \phi\left(x_{t s i} ;\left(\gamma_{t s i}\right)_{n}, L_{t s i}\right)}{\sum_{n=1}^{\infty} \phi\left(L_{t s i} ;\left(\gamma_{t s i}\right)_{n}, L_{t s i}\right)}
$$


where $i=1$ or 2 .

(viii) The final step is the combining of the trial-model prediction to produce a virtual model for comparison with the response of the beam in the physical space (e.g. $\left.w_{p s}=\beta_{1}^{-1} w_{t s 1}+R_{1}\left(\beta_{1}^{-1} w_{t s 1}-\beta_{2}^{-1} w_{t s 2}\right)\right)$ in accordance with Table 1.

The results of this study are presented in Fig. 7 with the deflection at the free end captured as function of time; there is perfect match between the virtual and physical results.

\subsection{Design III: First order with different beam materials}

The results of Design-III are depicted in Fig. 7 for the situation of completely different materials used for physical (steel) and trial-models 1 (aluminium) and 2 (copper); see Table 2 for properties. In Design-III, the applied forces at the free end in the physical and trial spaces are determined on the basis of the maximum yield stress in the outer fibres of each beam, i.e. on the basis of $F^{e n d}=2 Y I / L h$, where $Y$ is yield stress. These forces are used for initiation purposes and the determination of initial end deflections at static equilibrium. Following an identical procedure as discussed for Design II the following results are obtained: $g_{1}=0.51$, $g_{2}=0.27$ and $R_{1}=-0.78$. The results of the Design III study are presented in Fig. 7 with the deflection at the free end captured as function of time; there is again a perfect match between projected trial and physical results.

Table 2. Material properties of steel, aluminum and copper [29],[30],[31]

\begin{tabular}{|c|c|c|}
\hline Material & $\begin{array}{c}\text { Density: } \rho \\
\left(\mathrm{kg} / \mathrm{m}^{3}\right)\end{array}$ & $\begin{array}{c}\text { Young Modulus: } E \\
(\mathrm{GPa})\end{array}$ \\
\hline Steel (S355) & 7850 & 210 \\
\hline Aluminium & 2700 & 70 \\
\hline Copper & 8920 & 130 \\
\hline
\end{tabular}




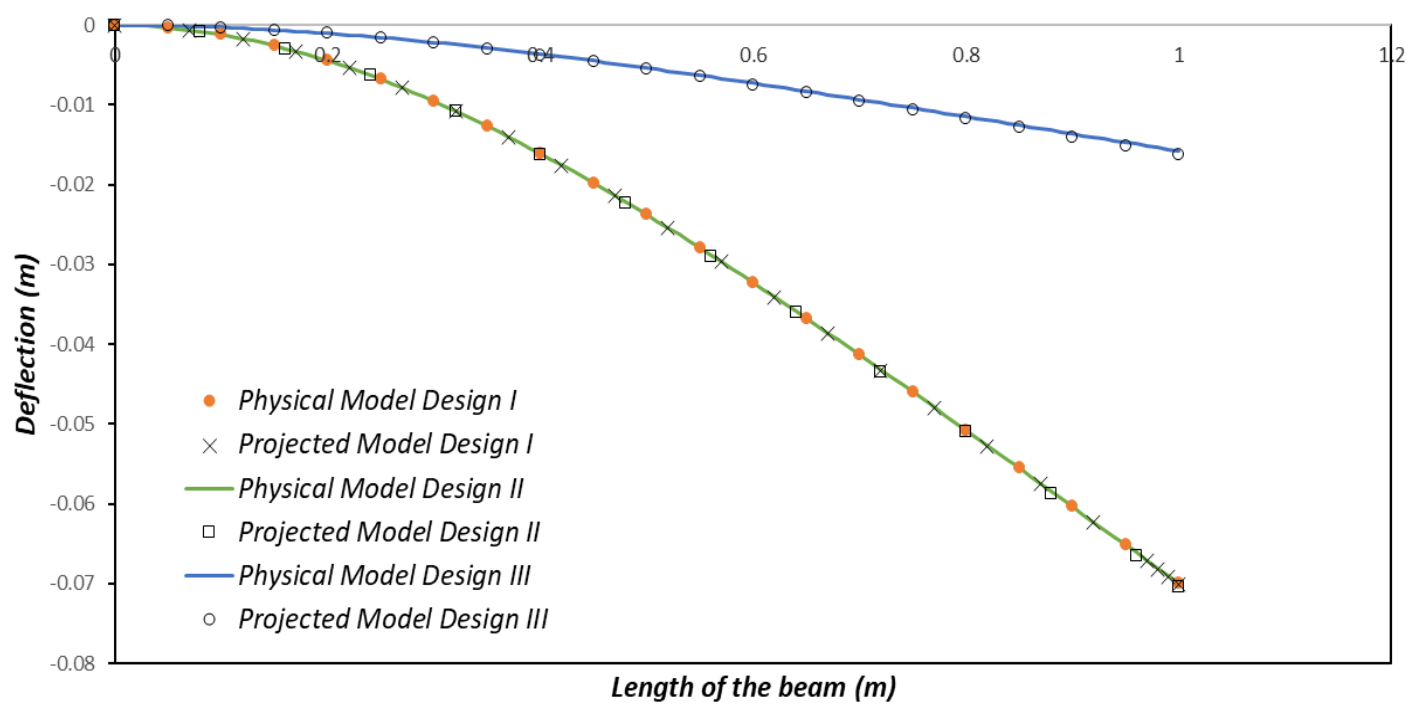

(a)

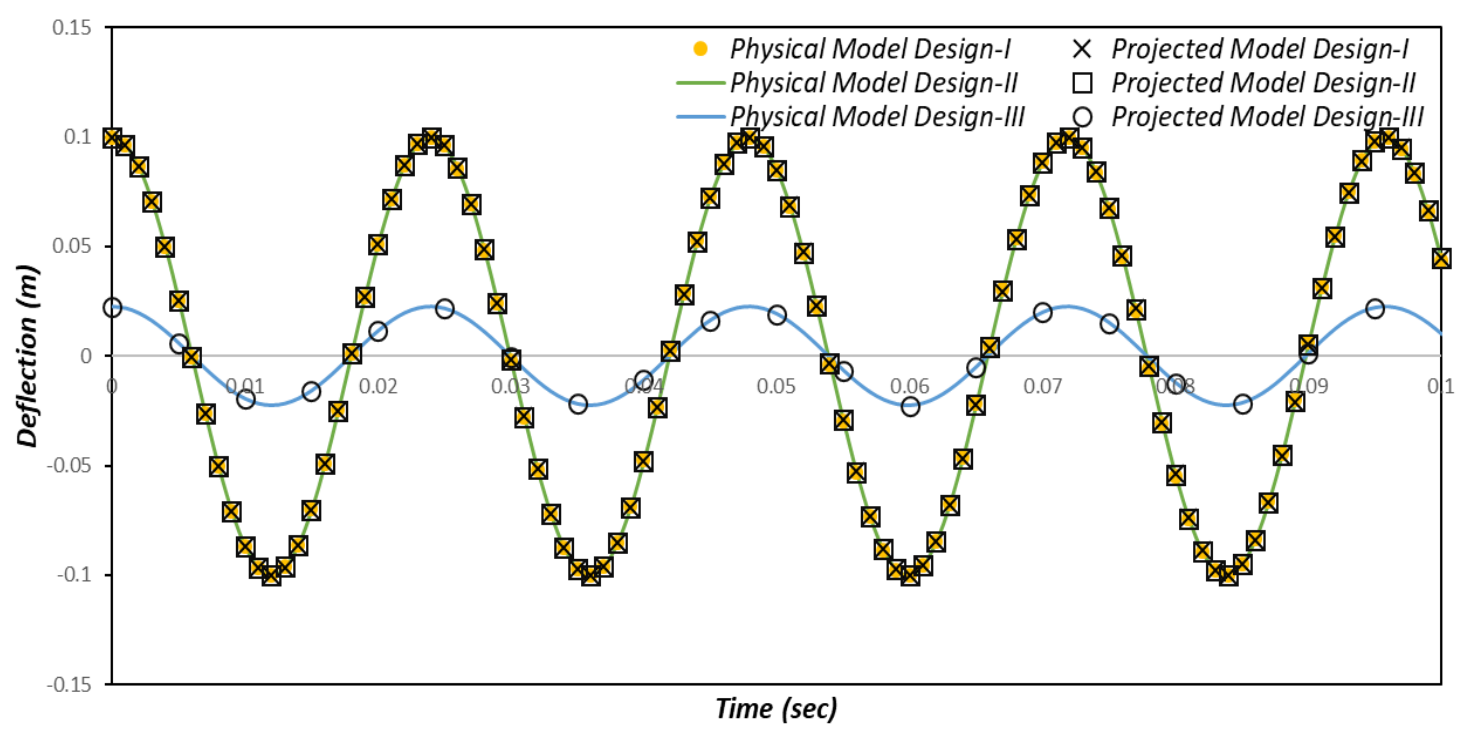

(b)

Figure 7. Predicting the (a) spatial $\left(t_{p s}=0.015 \mathrm{~s}\right)$ and (b) temporal response of the physical model using zeroth and first-order finite similitude theories in three designs.

\section{SEISMIC LOADING OF A COLUMN: NUMERICAL STUDY}

This section investigates the application of zeroth and first-order finite similitude to the earthquake loading of a relatively simple structure. A column is selected as the case study in order to focus the analysis on how the finite-similitude theory can be applied in seismic situations. Three possible column designs are considered for first order and two for zeroth order; each design is discussed in the subsections below. In order to give the study a degree 
of realism the Kocaeli Earthquake (1999) is applied in this study as the time-acceleration ground motion, which is depicted in Fig.8 [32].

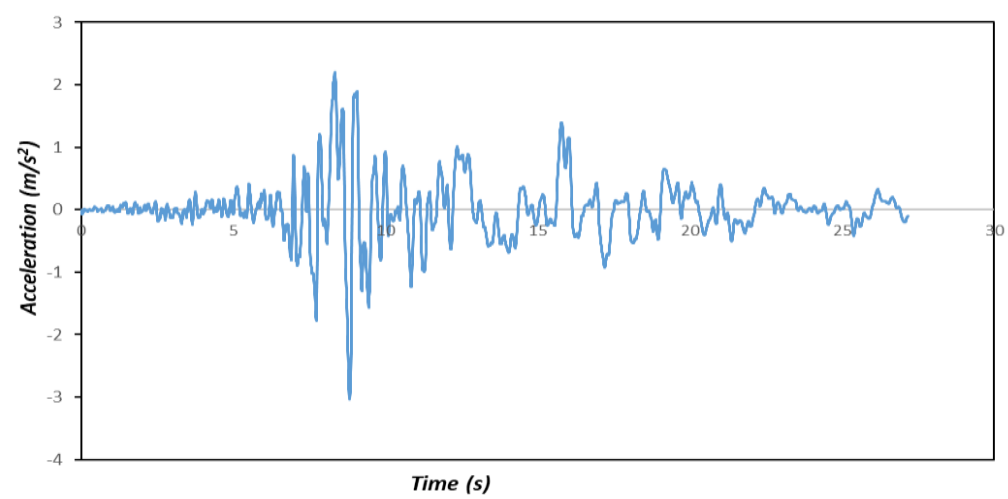

Figure 8. Acceleration - Time graph for Kocaeli earthquake.

\subsection{First-order Finite Similitude: steel column}

The application of the first-order finite similitude theory to a steel column housed in the physical space, where three combinations of different materials for trial models are examined. The purpose is to reveal how well the selected trial experiments capture the behaviour of the steel column. The dimensions of the steel column are provided in Fig. 9 and consist of a square section $a_{p s}=b_{p s}=0.1 \mathrm{~m}$ and height $l_{p s}=4 \mathrm{~m}$. Geometric dimensions of the trial models for both zeroth and first-order finite similitude depend on $\beta_{1}$ and $\beta_{2}$, which are set to $\beta_{1}=\frac{1}{4}$ and $\beta_{2}=\frac{1}{10}$. It is possible to let $\beta_{1}$ and $\beta_{2}$ remain unknown and determine them as part of the analysis but this aspect is not featured here as reasonable order-of-magnitude results are found possible on the basis of the selections made. As alluded in Appendix B, the determination of scaling parameters is based on what physical quantities are considered important to be targeted (e.g. stress, acceleration etc.) In seismic case studies the applied acceleration is an important physical quantity and matching applied acceleration (i.e. $\boldsymbol{A}_{p s}=\boldsymbol{A}_{t s 1}=\boldsymbol{A}_{t s 2}$ ) is often considered [33] as it can provide realistic and practical designs. Laboratories and devices for the application of seismic loads are of course limited in both size and load capacity and consequently constrain what is possible. 


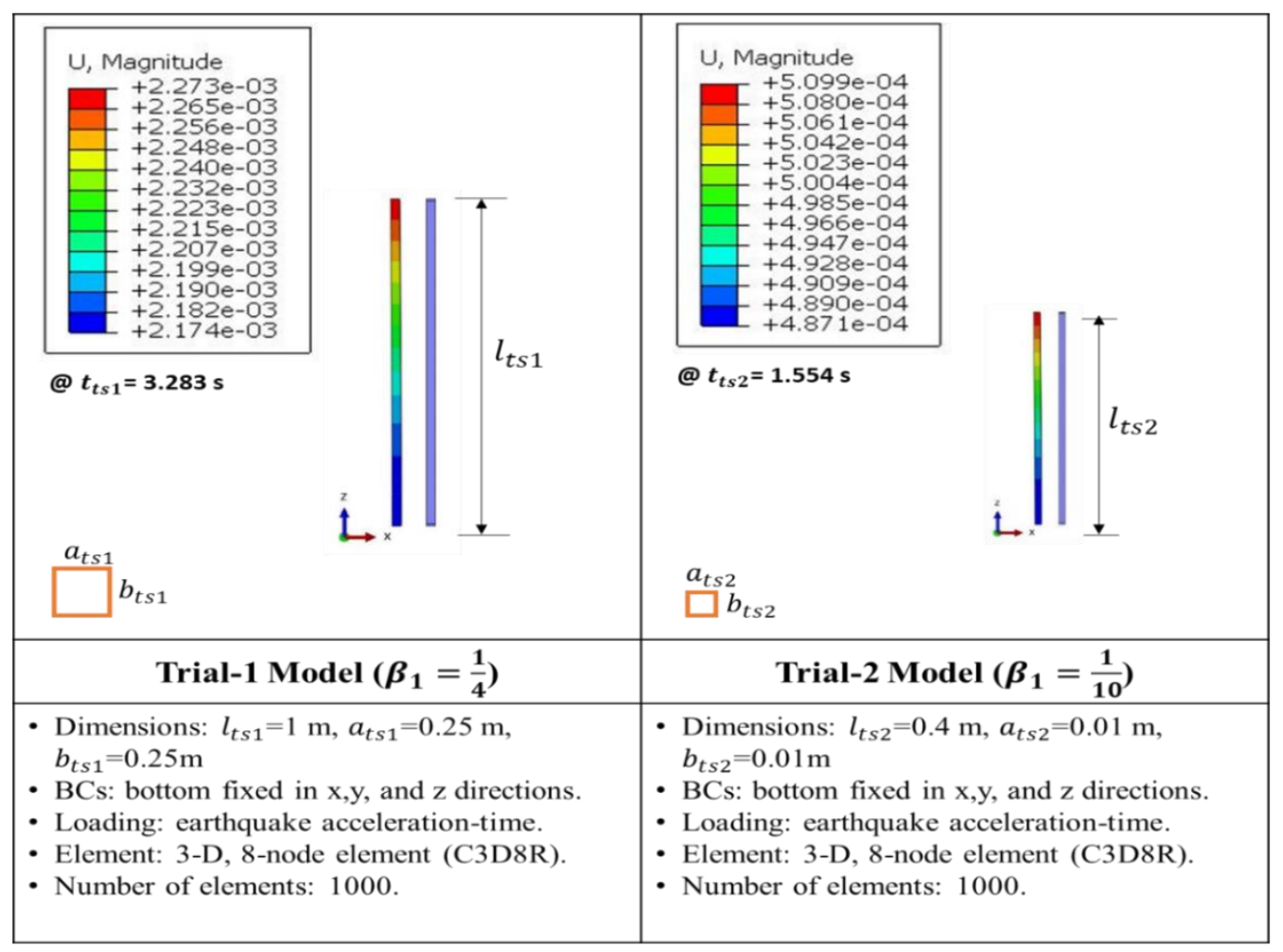

(a)

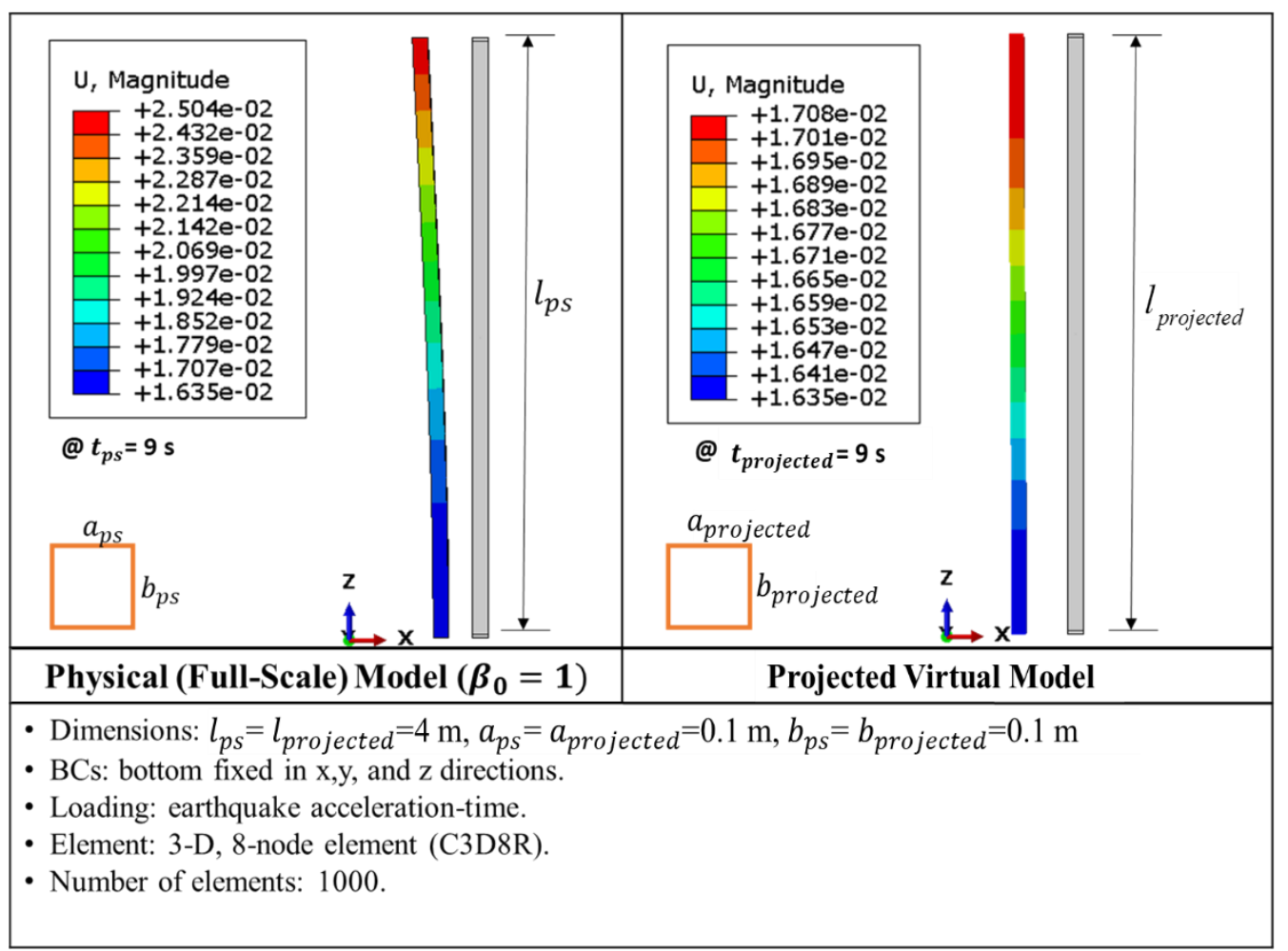

(b)

Figure 9. Deformed shapes in (a) trial space and (b) physical at synchronised times for 
As in Section 4, the problem reduces to finding the temporal scaling parameters $g_{1}$ and $g_{2}$ along with the first-order parameter $R_{1}$. Three equations are selected for this purpose which are:

$$
\begin{aligned}
& \boldsymbol{A}_{p s}=g_{1}^{2} \beta_{1}^{-1} \boldsymbol{A}_{t s 1}+R_{1}\left(g_{1}^{2} \beta_{1}^{-1} \boldsymbol{A}_{t s 1}-g_{2}^{2} \beta_{2}^{-1} \boldsymbol{A}_{t s 2}\right) \\
& \boldsymbol{\sigma}_{p s}=\alpha_{01}^{\rho} g_{1}^{2} \beta_{1} \sigma_{t s 1}+R_{1}\left(\alpha_{01}^{\rho} g_{1}^{2} \beta_{1} \sigma_{t s 1}-\alpha_{02}^{\rho} g_{2}^{2} \beta_{2} \sigma_{t s 2}\right) \\
& \boldsymbol{\varepsilon}_{p s}=\boldsymbol{\varepsilon}_{t s 1}+R_{1}\left(\boldsymbol{\varepsilon}_{t s 1}-\boldsymbol{\varepsilon}_{t s 2}\right)
\end{aligned}
$$

which are first-order approximations for acceleration, stress and strain, and where the expression for acceleration is readily derived by temporal differentiation of velocity in Eq. (17) and noting that $d t_{p s}=g_{1}^{-1} d t_{t s 1}=g_{2}^{-1} d t_{t s 2}$.

In all the tests considered the zeroth-order density is applied, i.e. $\rho_{p s}=\alpha_{01}^{\rho} \rho_{t s 1} \beta_{1}^{3}=\alpha_{02}^{\rho} \rho_{t s 2} \beta_{2}^{3}$, where $\alpha_{01}^{\rho}$ and $\alpha_{02}^{\rho}$ are set to match the density of the selected trial-space materials. The behaviour of both trial and physical-space columns is achieved numerically by means of the commercial finite-element software ABAQUS [34]; meshes and element type are depicted in Fig. 9. In practice of course, physical experiments would be undertaken but trialling the similitude theory is the focus here and numerical results are sufficient for this purpose.

\subsection{The proportional fields assumption}

In order to run scaled experiments in the trial space it is first necessary to specify $g_{1}$ and $g_{2}$, yet according to Eqs. (25), their solution is dependent on fields that are only available once the experiments have been run. To avoid the need for a time-consuming and somewhat impractical iterative approach to resolve this issue, a proportional-fields assumption is made. Recall that both dimensional analysis and zeroth-order finite similitude involve proportional fields, assumed ab initio for dimensional analysis and obtained as an output from finite similitude. The first-order theory on the other hand involves proportional differences as apparent in Table 1 for particular fields. With the knowledge that zeroth-order solutions are contained in first order (see Appendix B), it is a reasonable assumption therefore that the fields in Eqs. (25) are all proportional in the sense:

$$
\begin{aligned}
& \boldsymbol{A}_{t s 1}=\hat{a}_{1} \boldsymbol{A}_{p s} \\
& \boldsymbol{A}_{t s 2}=\hat{a}_{2} \boldsymbol{A}_{p s}
\end{aligned}
$$




$$
\begin{aligned}
& \sigma_{t s 1}=\hat{b}_{1} \sigma_{p s} \\
& \sigma_{t s 2}=\hat{b}_{2} \sigma_{p s} \\
& \boldsymbol{\varepsilon}_{t s 1}=\hat{c}_{1} \boldsymbol{\varepsilon}_{p s} \\
& \boldsymbol{\varepsilon}_{t s 2}=\hat{c}_{2} \boldsymbol{\varepsilon}_{p s},
\end{aligned}
$$

where it is understood that these relationships do not constrain the fields in Eqs. (25) as their purpose is only to aid the determination of $g_{1}, g_{2}$ and $R_{1}$, and where the hat "^" terms are non-zero dimensionless parameters.

Substitution of Eqs. (26) into Eqs. (25) provides

$$
\begin{aligned}
& 1=g_{1}^{2} \beta_{1}^{-1} \hat{a}_{1}+R_{1}\left(g_{1}^{2} \beta_{1}^{-1} \hat{a}_{1}-g_{2}^{2} \beta_{2}^{-1} \hat{a}_{2}\right) \\
& 1=\alpha_{01}^{\rho} g_{1}^{2} \beta_{1} \hat{b}_{1}+R_{1}\left(\alpha_{01}^{\rho} g_{1}^{2} \beta_{1} \hat{b}_{1}-\alpha_{02}^{\rho} g_{2}^{2} \beta_{2} \hat{b}_{1}\right) \\
& 1=\hat{c}_{1}+R_{1}\left(\hat{c}_{1}-\hat{c}_{2}\right)
\end{aligned}
$$

which can in principle be solved for $g_{1}, g_{2}$ and $R_{1}$ on specifying $\hat{a}_{i}, \hat{b}_{i}$ and $\hat{c}_{i}, i=1,2$.

To set the parameters $\hat{a}_{i}, \hat{b}_{i}$ and $\hat{c}_{i}$, considered here is the situation of a uniaxial rod in each space subjected to the same uniform acceleration and stretched to attain yield stress (i.e. $Y_{p s}$, $Y_{t s 1}$ and $Y_{t s 2}$ ) and yield strain (i.e. $\varepsilon_{p s}^{Y}=\frac{Y_{p s}}{E_{p s}}, \varepsilon_{t s 1}^{Y}=\frac{Y_{t s 1}}{E_{t s 1}}$ and $\varepsilon_{t s 2}^{Y}=\frac{Y_{t s 2}}{E_{t s 2}}$ ). This situation is possibly one of the simplest but substitution into Eqs. (26) for this case gives $\hat{a}_{1}=\hat{a}_{2}=1$, $\hat{b}_{1}=\frac{Y_{t s 1}}{Y_{p s}}, \hat{b}_{2}=\frac{Y_{t s 2}}{Y_{p s}}, \hat{c}_{1}=\frac{\varepsilon_{t s 1}^{Y}}{\varepsilon_{p s}^{Y}}$ and $\hat{c}_{2}=\frac{\varepsilon_{t s 2}^{Y}}{\varepsilon_{p s}^{Y}}$.

\subsection{Application of the theory}

Three designs are considered, where are labelled Design I, Design II and Design III, the details of which are provided in Table 3. Design I is the case where the same grade of steel (i.e. S355 [26]) is used in all spaces for the columns. Design II applies different grades of steel (see [28] and [29]) in each space as indicated in Table 3. Design III on the other hand again uses different materials with different grades of steel in the physical and trial-space one but aluminium for trial-space two.

For all three designs the applied acceleration $A_{1}$ in $\mathrm{x}$-direction is the same and the yield stresses $\left(Y_{p s}, Y_{t s 1}\right.$ and $\left.Y_{t s 2}\right)$ and yield strains $\left(\varepsilon_{p s}^{Y}, \varepsilon_{t s 1}^{Y}\right.$ and $\left.\varepsilon_{t s 2}^{Y}\right)$ for the three designs can be found in Table 3. A feature of Design I is that $\hat{c}_{1}=\hat{c}_{2}$, which removes Eq. (27c). The two 
remaining equations Eq. (27a) and Eq. (27a) have too many unknowns and to resolve the situation, so the zeroth-order condition $1=g_{1}^{2} \beta_{1}^{-1}$ (i.e. first two terms in Eq. (27a)) is assumed to apply, which gives $g_{1}=\sqrt{\beta_{1}}=\frac{1}{2}$. The remaining values (i.e. $g_{2}$ and $R_{1}$ ), calculated using Eq. (27a) and (27b), can be found in Table 3. Qualitative results for the distribution of the displacement magnitude are provided in Fig. 9. along with a detailed model description. Design II involves three grades of steel [28] provides values $g_{1}=0.365, g_{2}=0.173$ and $R_{1}=2$ on solution of Eqs. (27). Design III on the other hand did not provide a solution to Eqs. (27), so was resolved in the same manner as Design I, i.e. by setting $g_{1}=\sqrt{\beta_{1}}=\frac{1}{2}$ and solving for $g_{2}$ and $R_{1}$, which provided $g_{2}=0.316$, and $R_{1}=0.5$ as recorded in Table 3 . With the determination of $g_{1}, g_{2}$ and scaling parameters, the trial models result results were obtained, projected to the full scale and combined by means of the first-order theory. The results for the top-story drift of the column for all three designs are presented in Fig. 10. Show in the figure are the virtual results obtained on application of the first-order theory along with those determined by virtue of direct simulation of the full-scale model in the physical space.

Table 3. Material properties of three models and calculated time scaling and values.

\begin{tabular}{|c|c|c|c|c|c|c|c|c|c|}
\hline $\begin{array}{c}\text { Design } \\
\mathrm{s}\end{array}$ & Models & $\begin{array}{c}\text { Materia } \\
1\end{array}$ & $\begin{array}{l}\text { Density } \\
\left(\mathrm{kg} / \mathrm{m}^{3}\right)\end{array}$ & $\begin{array}{c}\text { Young's } \\
\text { Modulus } \\
\left(\mathbf{1 0}^{11} \mathbf{P a}\right)\end{array}$ & $\begin{array}{c}\text { Yield } \\
\text { Stress } \\
\left(\mathbf{1 0}^{\mathbf{8}} \mathbf{P a}\right)\end{array}$ & $\begin{array}{l}\text { Yield } \\
\text { Strain } \\
\left(1^{-3}\right)\end{array}$ & $g_{1}$ & $g_{2}$ & $R_{1}$ \\
\hline \multirow{3}{*}{$\begin{array}{c}\text { Design } \\
\text { I }\end{array}$} & $\begin{array}{c}\text { Full-scale } \\
\text { Model }\end{array}$ & S355 & 7850 & 2.1 & 3.55 & 1.69 & \multirow{3}{*}{0.5} & \multirow{3}{*}{0.316} & \multirow{3}{*}{0.5} \\
\hline & $\begin{array}{l}\text { Trial-1 } \\
\text { Model }\end{array}$ & S355 & 7850 & 2.1 & 3.55 & 1.69 & & & \\
\hline & $\begin{array}{l}\text { Trial-2 } \\
\text { Model }\end{array}$ & S355 & 7850 & 2.1 & 3.55 & 1.69 & & & \\
\hline \multirow{3}{*}{$\begin{array}{c}\text { Design } \\
\text { II }\end{array}$} & $\begin{array}{c}\text { Full-scale } \\
\text { Model }\end{array}$ & S355 & 7850 & 2.1 & 3.55 & 1.69 & \multirow{3}{*}{0.365} & \multirow{3}{*}{0.173} & \multirow{3}{*}{2} \\
\hline & $\begin{array}{l}\text { Trial-1 } \\
\text { Model }\end{array}$ & S275 & 7850 & 2.1 & 2.75 & 1.31 & & & \\
\hline & $\begin{array}{l}\text { Trial-2 } \\
\text { Model }\end{array}$ & S235 & 7850 & 2.1 & 2.35 & 1.12 & & & \\
\hline \multirow{3}{*}{$\begin{array}{l}\text { Design } \\
\text { III }\end{array}$} & $\begin{array}{c}\text { Full-scale } \\
\text { Model }\end{array}$ & S355 & 7850 & 2.1 & 3.55 & 1.69 & \multirow{3}{*}{0.5} & \multirow{3}{*}{0.316} & \multirow{3}{*}{$\begin{array}{c}0.08 \\
82\end{array}$} \\
\hline & $\begin{array}{l}\text { Trial-1 } \\
\text { Model } \\
\end{array}$ & S275 & 7850 & 2.1 & 2.75 & 1.31 & & & \\
\hline & $\begin{array}{l}\text { Trial-2 } \\
\text { Model }\end{array}$ & $\mathrm{Al}$ & 2770 & 0.7 & 3.37 & 4.814 & & & \\
\hline
\end{tabular}




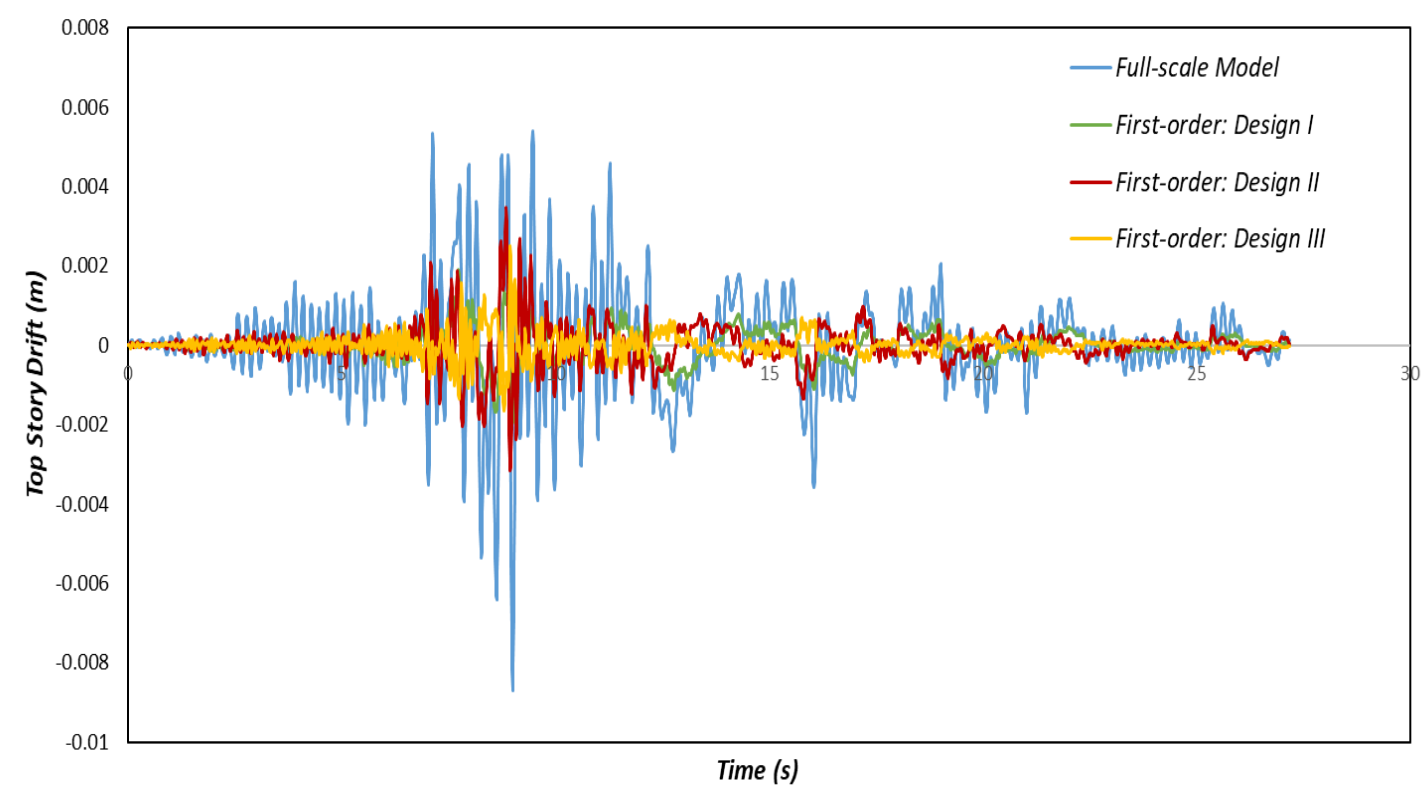

Figure 10. First-order predicts and direct-full scale simulation

It is apparent on examination of Fig. 10 that the different designs provide different outputs and there are also in place significant differences between the virtual-model predictions and the direct full-scale simulation. It is possibly not too surprising that the scaled models were unable to fully capture the precise full-scale behaviour of an earthquake event. However, considered in the next section, are single-trial space models to better highlight the significant improvement achieved by the new theory.

\subsection{Zeroth-order finite similitude design}

Each of the trial models at scales $\beta_{1}=\frac{1}{4}$ and $\beta_{2}=\frac{1}{10}$ (introduced in Section 5.3) are reconsidered here using the zeroth-order theory, hence only one trial model per analysis. The zeroth order relationship $\boldsymbol{A}_{p s}=g^{2} \beta^{-1} \boldsymbol{A}_{t s}$ with proportionality gives $1=g^{2} \beta^{-1} \hat{a}$ and on setting $\hat{a}=1$ provides $g=\sqrt{\beta}$. Other zeroth-order scaling identities are provided in Table. 1 . For the trial models that same materials as in Section 5.3 are used and details are provided in Table 5. Note that two test cases are considered (test cases I and II) with each consisting of two trial models (trial models I and II) corresponding to the two scales (i.e. $\beta_{1}=\frac{1}{4}$ and $\beta_{2}=\frac{1}{10}$ ). The projected models correspond to the projection of the trial models into the physical space and the salient projected material properties are tabulated in Table 4. Shown in Fig. 11 is the temporal response of top displacement, which is recognised to be important for the comparison of building models under seismic loading. 
Table 4. Zeroth-order Finite Similitude scaling parameters and material properties

\begin{tabular}{|c|c|c|c|c|c|c|c|c|}
\hline $\begin{array}{c}\text { Test } \\
\text { Cases }\end{array}$ & Models & & $\begin{array}{l}\text { Density } \\
\left(\mathrm{kg} / \mathrm{m}^{3}\right)\end{array}$ & $\begin{array}{l}\text { Young's } \\
\text { Modulus } \\
\left(1^{11} \mathrm{~Pa}\right)\end{array}$ & $\begin{array}{c}\text { Yield } \\
\text { Stress } \\
\left(1^{8} \mathbf{P a}\right)\end{array}$ & $\beta$ & $\alpha_{01}^{\rho}$ & $g=\sqrt{\beta}$ \\
\hline \multirow{4}{*}{$\begin{array}{c}\text { Test } \\
\text { Case I }\end{array}$} & Trial Model 1 & S355 & 7850 & 2.1 & 3.55 & $1 / 4$ & 64 & 2 \\
\hline & Trial Model 2 & S355 & 7850 & 2.1 & 3.55 & $1 / 10$ & 1000 & $\sqrt{10}$ \\
\hline & Projected Model-1 & & 7850 & 8.4 & 14.2 & & & \\
\hline & Projected Model-2 & & 7850 & 210 & 35.5 & & & \\
\hline \multirow{4}{*}{$\begin{array}{c}\text { Test } \\
\text { Case } \\
\text { II }\end{array}$} & Trial Model 1 & S275 & 7850 & 2.1 & 2.75 & $1 / 4$ & 64 & 2 \\
\hline & Trial Model 2 & S235 & 7850 & 2.1 & 2.35 & $1 / 10$ & 1000 & $\sqrt{10}$ \\
\hline & Projected Model 1 & & 7850 & 8.4 & 11 & & & \\
\hline & Projected Model 2 & & 7850 & 210 & 23.5 & & & \\
\hline
\end{tabular}

The graphs in Fig. 11(b) illustrate the marked improvement achievable on application of the first-order finite similitude theory. The results for first-order Design I are much improved over those corresponding to Case I for both trial modes. Likewise, first-order Design II outperforms all case and although fails to capture all aspects of the full-scale simulation it is vastly superior to single-space trial models. The advantage offered by first-order finite similitude theory in seismic studies is that it provides an ability to fix not only acceleration but also other material properties. Zeroth order is limited in only enabling the fixing of acceleration and as revealed in Table 4, with other material properties not matched.

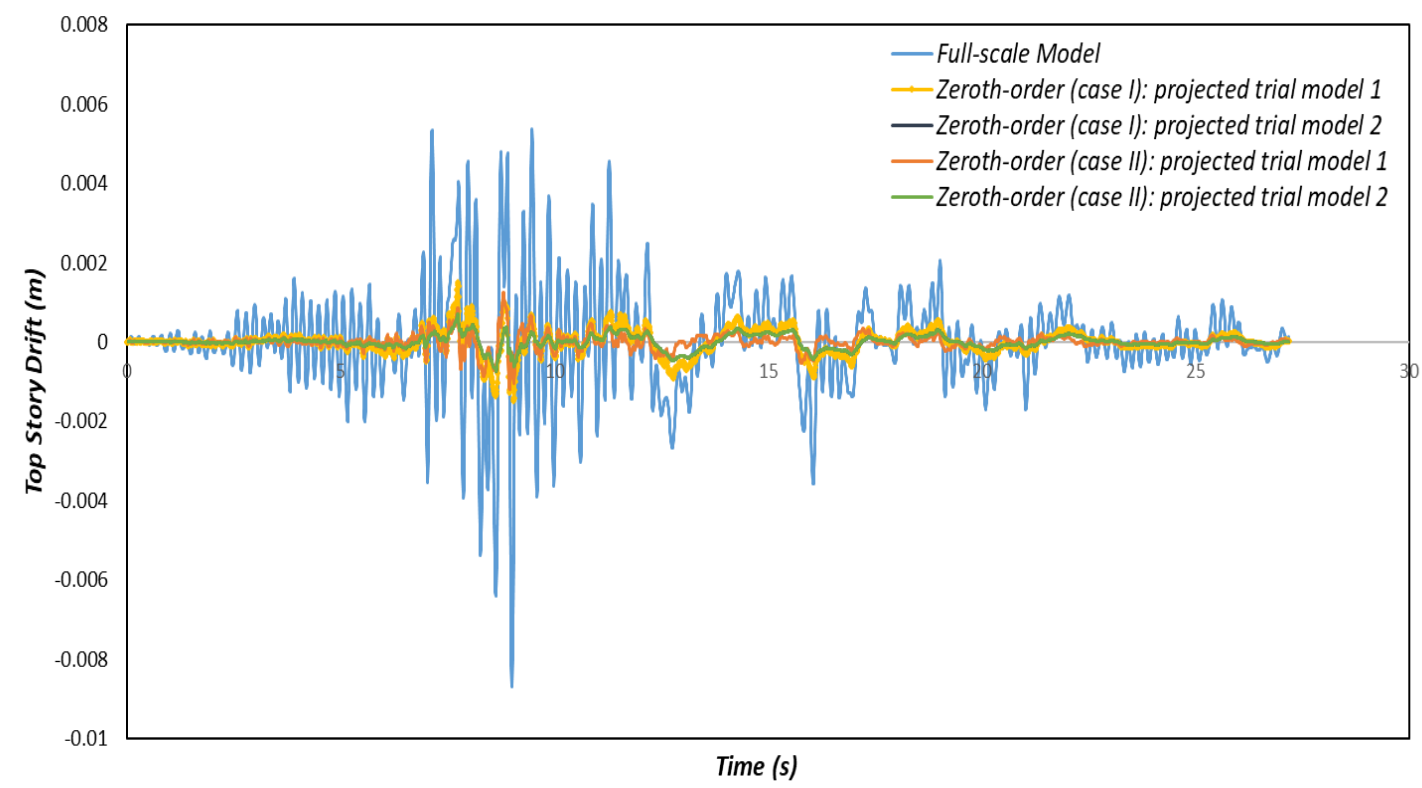

(a) 


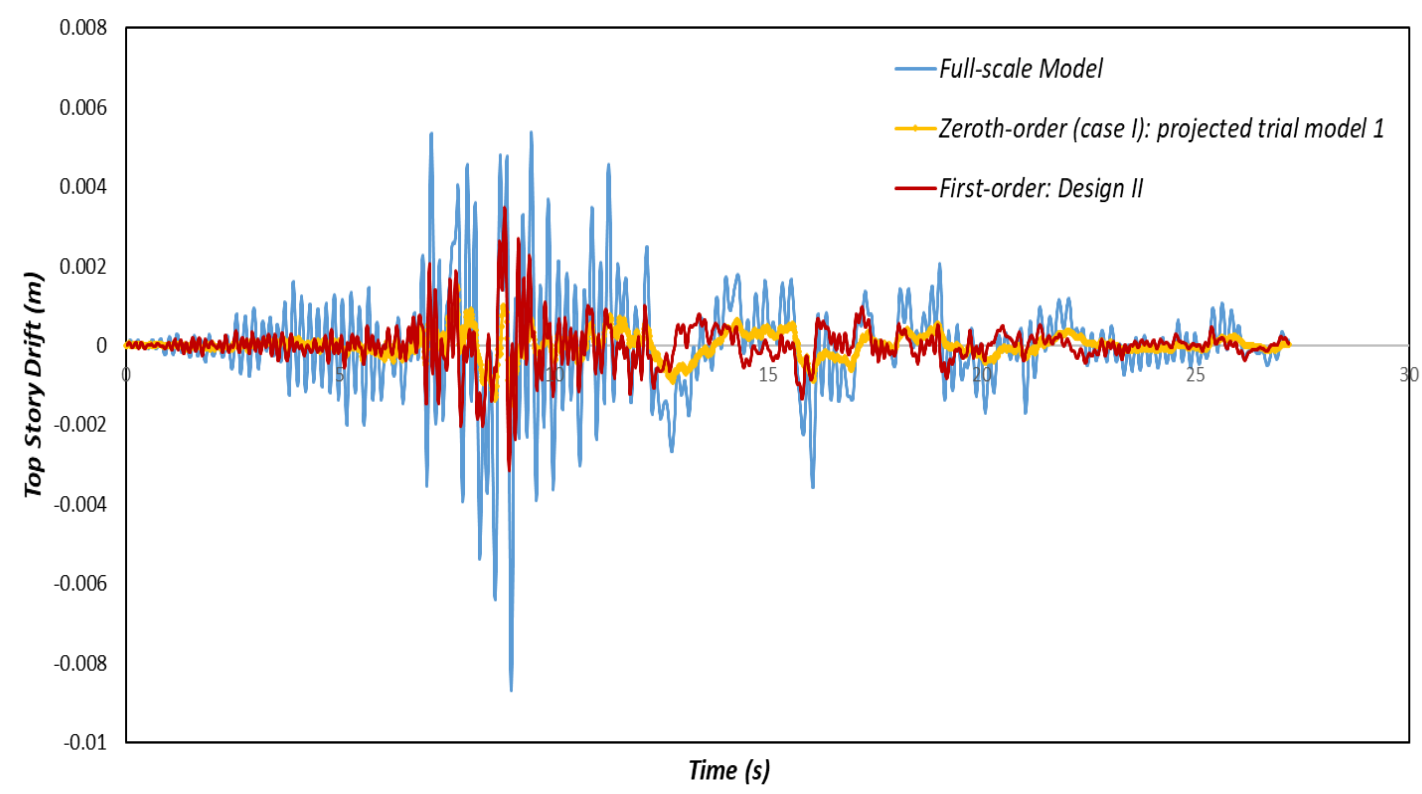

(b)

Figure 11. Performance of zeroth and first-order finite similitude designs

To demonstrate the practical applicability of the new theory a more realistic structure is examined in the next section.

\section{MULTI-STOREY FRAME EXPOSED TO CYCLIC LOADING: A NUMERICAL STUDY}

Different forms of seismic or environmental loading can impact on the performance of a structure. Because of the inherent uncertainty associated with these types of load it is common practice in models to load systems quasi-statically with consistently increasing loads. Although recognising that this approach does have its limitations [34] engineering structures must have the capacity to resist thousands of loading cycles to withstand disasters such as earthquakes [35]. Cyclic thermal and mechanical loads can push systems past the elastic region wherein plastic straining occurs [36] and it is important therefore that systems remain safe and serviceable under such conditions.

It is of interest therefore to examine the behaviour of structures under cyclic loading and for this reason, a steel frame consisting of two bays and three storey is modelled and the analysis executed by applying cyclic displacement loads at the top corner of the structure. The beam and column cross-sections are HSS $127 \mathrm{~mm} \times 127 \mathrm{~mm} \times 9.5 \mathrm{~mm}$ and the storey height is $0.8 \mathrm{~m}$ while the bay is $1.25 \mathrm{~m}$ [37]. Scaled models are also created to test out the ability of scaling to capture in this case the effects of cyclic loading on a building structure. A schematic diagram is presented in Fig. 12 showing the real full-scale and trial models along with the projected trial models and their combination to form the full-scale virtual model. 
The scales selected for the study are $\beta_{1}=\frac{1}{4}$ and $\beta_{2}=\frac{1}{6}$ as indicated in Fig. 12. Both zeroth and first-order theory is applied, and details are presented in Tables 5 and 6, respectively. Analysis of the full-scale and trial models is performed model using Abaqus explicit and for consistency a transient-cyclic analysis is performed.

Table 5. Zeroth-order material properties and scaling parameters

\begin{tabular}{|c|c|c|c|c|c|c|c|c|}
\hline Design & Model & Mat. & $\begin{array}{c}\text { Density } \\
\left(\mathbf{k g} / \mathbf{m}^{\mathbf{3}}\right)\end{array}$ & $\begin{array}{c}\text { Young's } \\
\text { Modulus } \\
\left(\mathbf{1 0}^{\mathbf{1 1}} \mathbf{P a}\right)\end{array}$ & $\begin{array}{c}\text { Yield } \\
\text { Stress } \\
\left(\mathbf{1 0}^{\mathbf{8}} \mathbf{P a}\right)\end{array}$ & $\beta$ & $\alpha^{\rho}$ & $g$ \\
\hline \multirow{4}{*}{$\begin{array}{c}\text { Design } \\
\text { I }\end{array}$} & Trial Model 1 & $\mathrm{S} 235$ & 7850 & 2.1 & 2.35 & $1 / 4$ & 64 & 0.30727 \\
\cline { 2 - 10 } & Trial Model 2 & $\mathrm{Al}$ & 2770 & 0.7 & 3.37 & $1 / 4$ & 181 & 0.15242 \\
\cline { 2 - 10 } & $\begin{array}{c}\text { Projected } \\
\text { Model 1 }\end{array}$ & & 7850 & 3.17 & 3.55 & & & \\
\cline { 2 - 10 } & $\begin{array}{c}\text { Projected } \\
\text { Model 2 }\end{array}$ & & 7850 & 0.737 & 3.55 & & & \\
\hline \multirow{3}{*}{$\begin{array}{c}\text { Design } \\
\text { II }\end{array}$} & Trial Model 1 & $\mathrm{S} 235$ & 7850 & 2.1 & 2.35 & $1 / 4$ & 64 & 0.25 \\
\cline { 2 - 10 } & Trial Model 2 & $\mathrm{Al}$ & 2770 & 0.7 & 3.37 & $1 / 4$ & 181 & 0.257 \\
\cline { 2 - 10 } & $\begin{array}{c}\text { Projected } \\
\text { Model 1 }\end{array}$ & & 7850 & 2.1 & 2.35 & & & \\
\cline { 2 - 10 } & $\begin{array}{c}\text { Projected } \\
\text { Model 2 }\end{array}$ & & 7850 & 2.1 & 10.1 & & & \\
\hline
\end{tabular}

The material used in the full-scale structure is steel S355 and the targeted-physical quantities for the projected trial models are density and yield stress and/or Young's modulus. For zeroth order this involves the relationships $\rho_{p s}=\alpha_{01}^{\rho} \rho_{t s 1} \beta_{1}^{3}$ and $Y_{p s}=\alpha_{01}^{\rho} g_{1}^{2} \beta_{1} Y_{t s 1}$ or $E_{p s}=\alpha_{01}^{\rho} g_{1}^{2} \beta_{1} E_{t s 1}$, respectively. With $\beta_{1}=\frac{1}{4}$ the values of $\alpha_{01}^{\rho}$ and $g_{1}$ can be determined and their values are provided in Table 5 for the different material combinations. As regards firstorder, the proportional method of Section 5.2 is applied leading to the equations

$$
\begin{aligned}
& 1=\alpha_{01}^{\rho} g_{1}^{2} \beta_{1} \hat{b}_{1}+R_{1}\left(\alpha_{01}^{\rho} g_{1}^{2} \beta_{1} \hat{b}_{1}-\alpha_{02}^{\rho} g_{2}^{2} \beta_{2} \hat{b}_{1}\right) \\
& 1=\alpha_{01}^{\rho} g_{1}^{2} \beta_{1} \hat{e}_{1}+R_{1}\left(\alpha_{01}^{\rho} g_{1}^{2} \beta_{1} \hat{e}_{1}-\alpha_{02}^{\rho} g_{2}^{2} \beta_{2} \hat{e}_{2}\right)
\end{aligned}
$$

arising for stress and stiffness considerations with $\hat{b}_{1}=\frac{Y_{s 1}}{Y_{p s}}, \hat{b}_{2}=\frac{Y_{t s 2}}{Y_{p s}}, \hat{e}_{1}=\frac{E_{t s 1}}{E_{p s}}$ and $\hat{e}_{2}=\frac{E_{t s 2}}{E_{p s}}$, and in order to solve these two equations for $g_{2}$ and $R_{1}$, it is necessary to set $g_{1}$.

This is done by means of zeroth-order theory and the consequential values of $g_{2}$ and $R_{1}$ obtained from Eqs. (28) are provided in Table 6. Note the common values of $g_{1}$ in Tables 5 and 6 indicating that Design $\mathrm{I}$ is the design taken forward to first-order analysis. The boundary and loading for each of the full scale and trial models are presented in Fig. 13 along with mesh details for the analysis. 


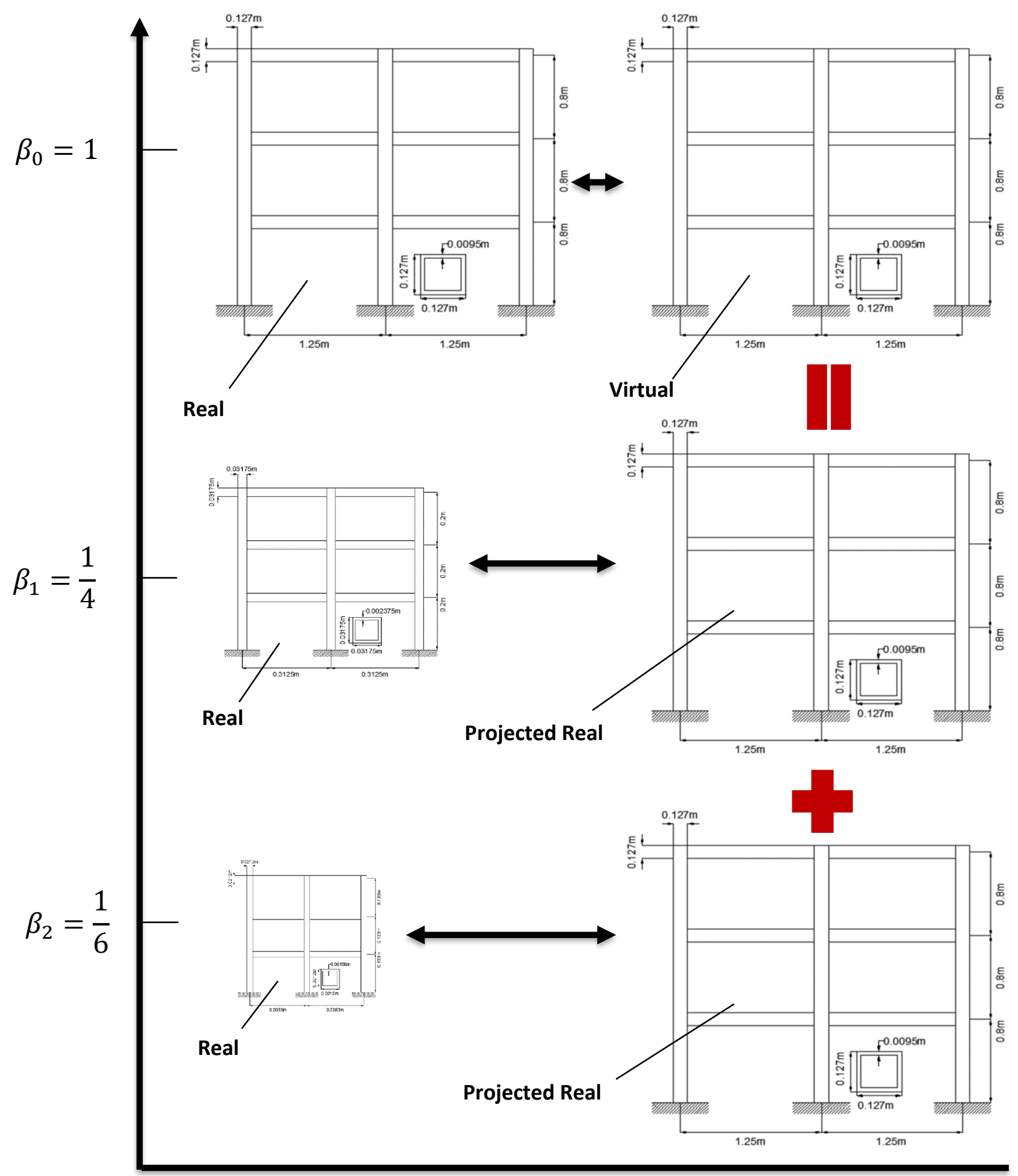

Figure 12. Scaled models for a two-bay, three-storey structure 
Table 6. First-order material properties and scaling parameters

\begin{tabular}{|c|c|c|c|c|c|c|c|c|}
\hline $\begin{array}{c}\text { Design } \\
\mathrm{s}\end{array}$ & & Materials & $\begin{array}{l}\text { Density } \\
\left(\mathrm{kg} / \mathrm{m}^{3}\right)\end{array}$ & $\begin{array}{l}\text { Young's } \\
\text { Modulus } \\
\left(1^{11} \mathrm{~Pa}\right)\end{array}$ & $\begin{array}{c}\text { Yield } \\
\text { Stress } \\
\left(10^{6} \quad \mathrm{~Pa}\right)\end{array}$ & $g_{1}$ & $g_{2}$ & $R_{1}$ \\
\hline \multirow{3}{*}{$\begin{array}{c}\text { Design } \\
\text { I }\end{array}$} & $\begin{array}{c}\text { Full-scale } \\
\text { Model }\end{array}$ & S355 & 7850 & 2.1 & 3.55 & \multirow{3}{*}{0.3073} & \multirow{3}{*}{0.1894} & \multirow{3}{*}{-2.324} \\
\hline & $\begin{array}{c}\text { Trial Model } \\
1\end{array}$ & S235 & 7850 & 2.1 & 2.35 & & & \\
\hline & $\begin{array}{c}\text { Trial Model } \\
2\end{array}$ & S275 & 7850 & 2.1 & 2.75 & & & \\
\hline \multirow{3}{*}{$\begin{array}{c}\text { Design } \\
\text { II }\end{array}$} & $\begin{array}{c}\text { Full-scale } \\
\text { Model }\end{array}$ & S355 & 7850 & 2.1 & 3.55 & \multirow{3}{*}{0.153} & \multirow{3}{*}{0.205} & \multirow{3}{*}{-0.845} \\
\hline & $\begin{array}{c}\text { Trial Model } \\
1\end{array}$ & $\mathrm{Al}$ & 2770 & 0.7 & 3.37 & & & \\
\hline & $\begin{array}{c}\text { Trial Model } \\
2\end{array}$ & $\mathrm{~S} 235$ & 7850 & 2.1 & 2.35 & & & \\
\hline
\end{tabular}

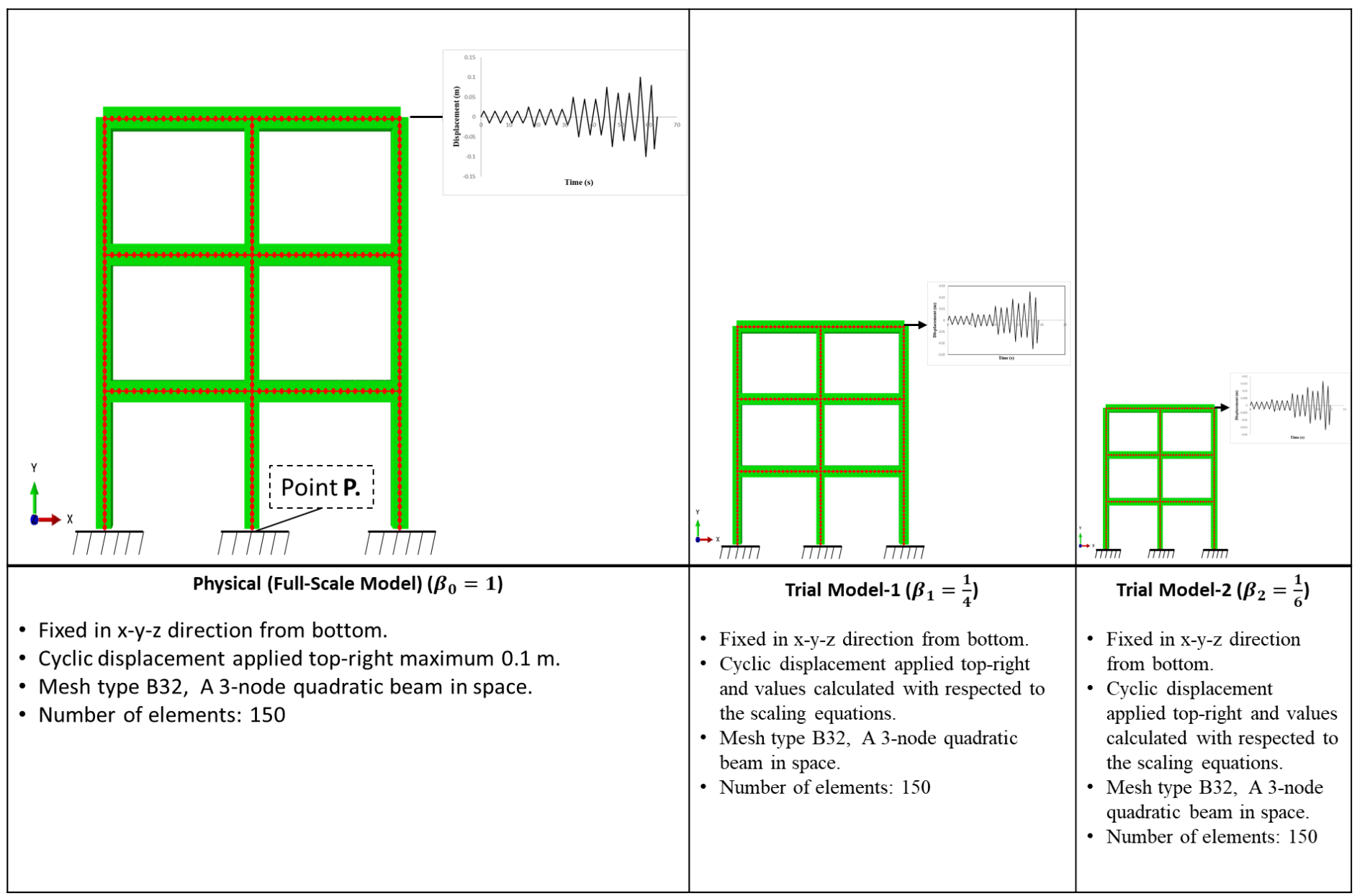

Figure 13. Boundary and loading for physical and trial models 
The mesh densities are those obtained from a mesh-sensitivity study and provide converged solutions. The loading applied in this study consists of a cyclically load generally increasing with amplitude as time progresses as shown in Figs. 13 and 14 (see references [36] and [37] for details). The results from the mesh-sensitivity study under the loading depicted in Fig. 14 are presented in Fig. 15. The mesh size/number of elements had negligible influence on the results obtained. Note that the choice of loading is similar to that of an earthquake with increasing amplitudes with time. It can be seen from Fig. 15 that the loading consists of a form of generally increasing sharp waves punctuated by periods of constant amplitudes.

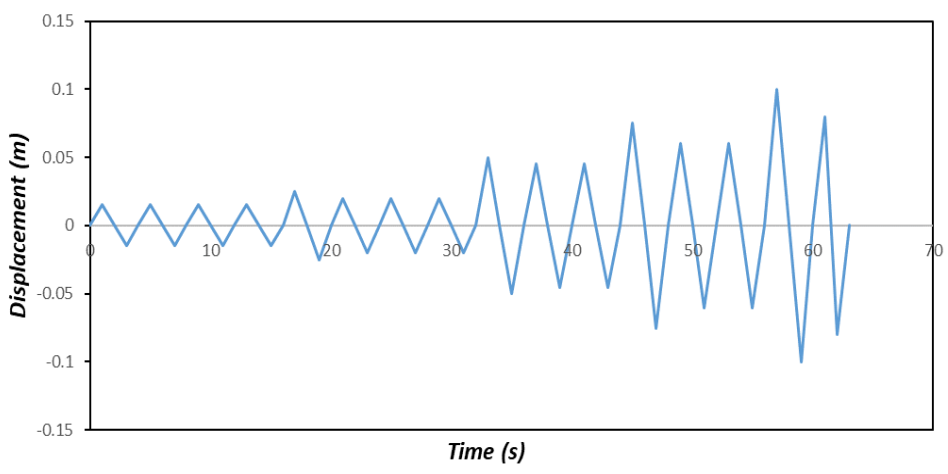

Figure 14. Applied cyclic displacement-time graph (based on [38]).

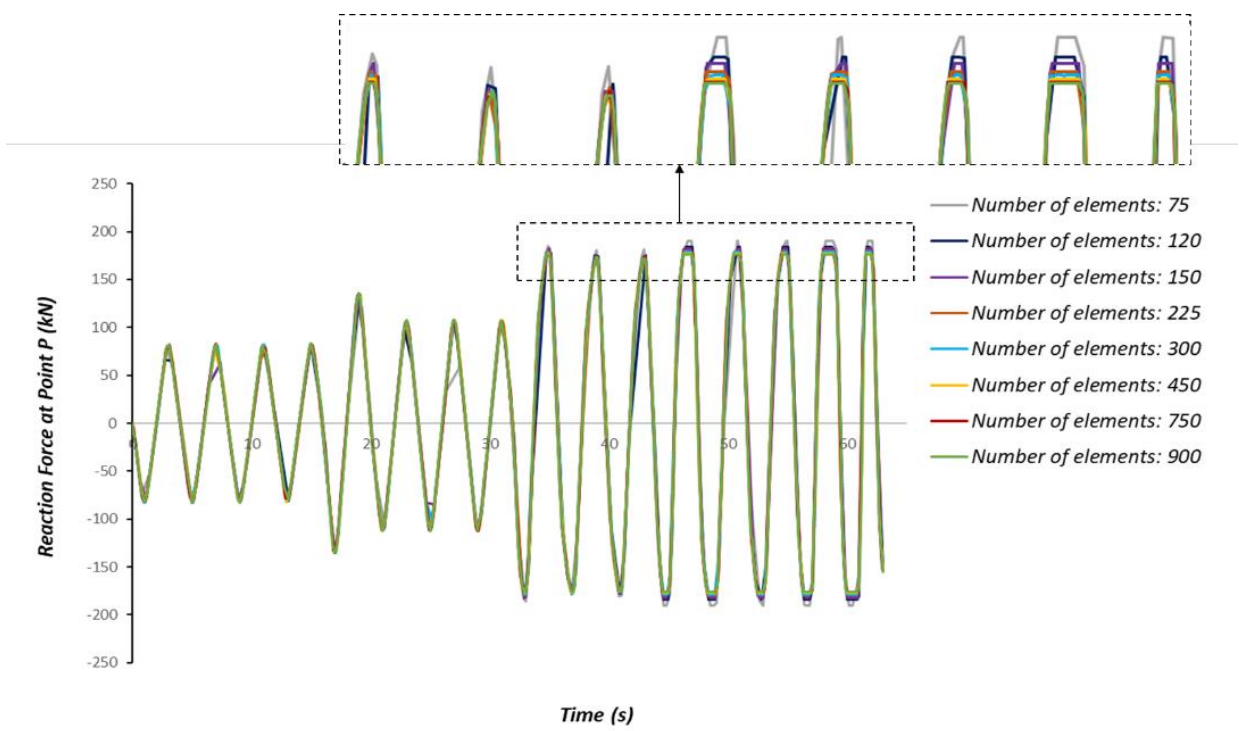

Figure 15. Mesh sensitivity analysis at point $\mathrm{P}$ shown in Fig. 13.

The results of the study are presented in Figs. 16 to 19, with zeroth-order results presented in Figs. 16 and 17. The results of Figs. 16 and 17 indicate that Design I (Trial 1) as defined in Table 6 provides the best approximation from the cases considered. Examination of the values for yield stress, density and Young's modulus for the projected model indicate the reason for this, since these are closest to those of the full-scale model. However, the match is not perfect as indicated by the inclined portion of the graph which does not coincide. 


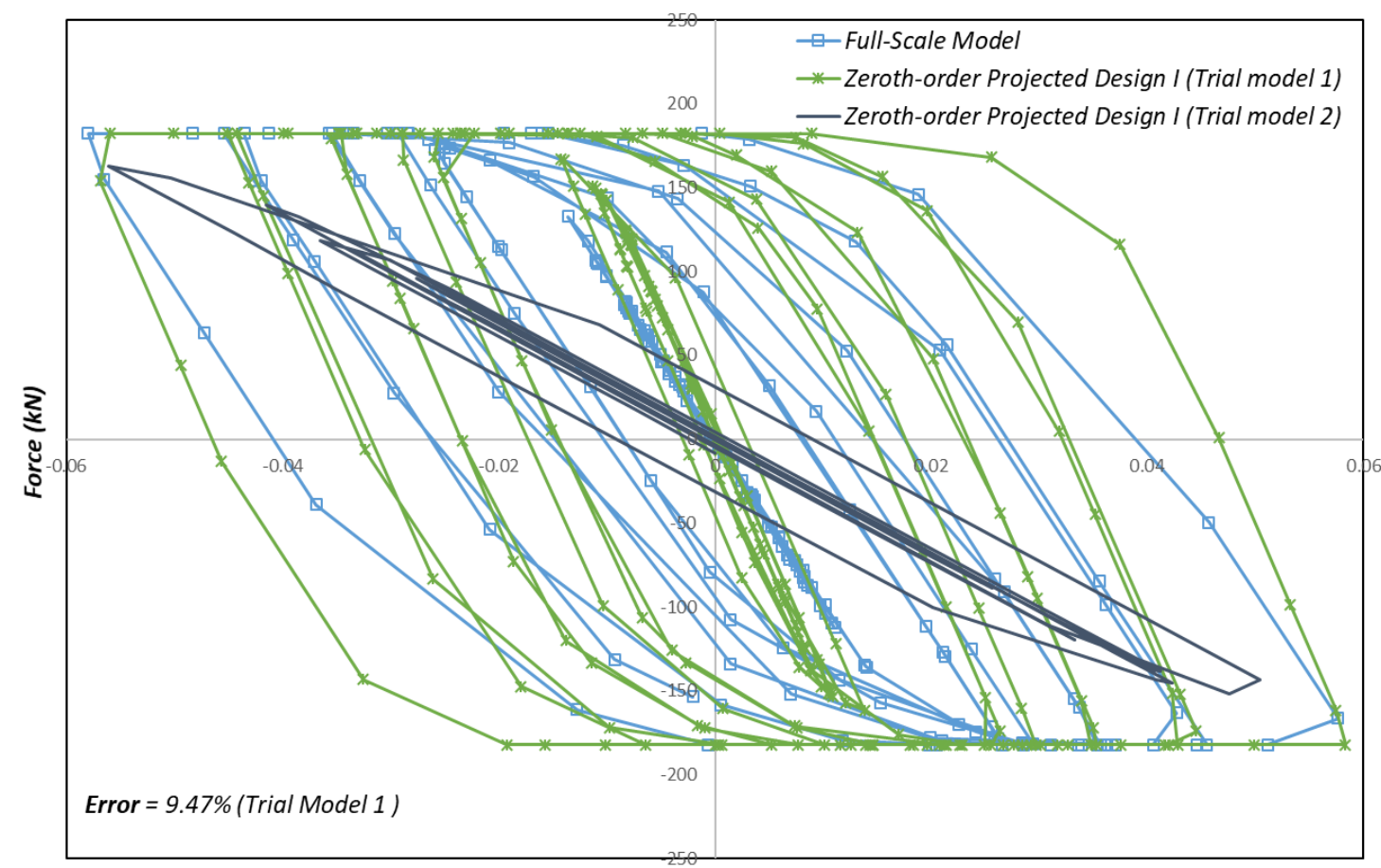

Displacement ( $m$ )

Figure 16. Zeroth order cyclic analysis of Design I models.

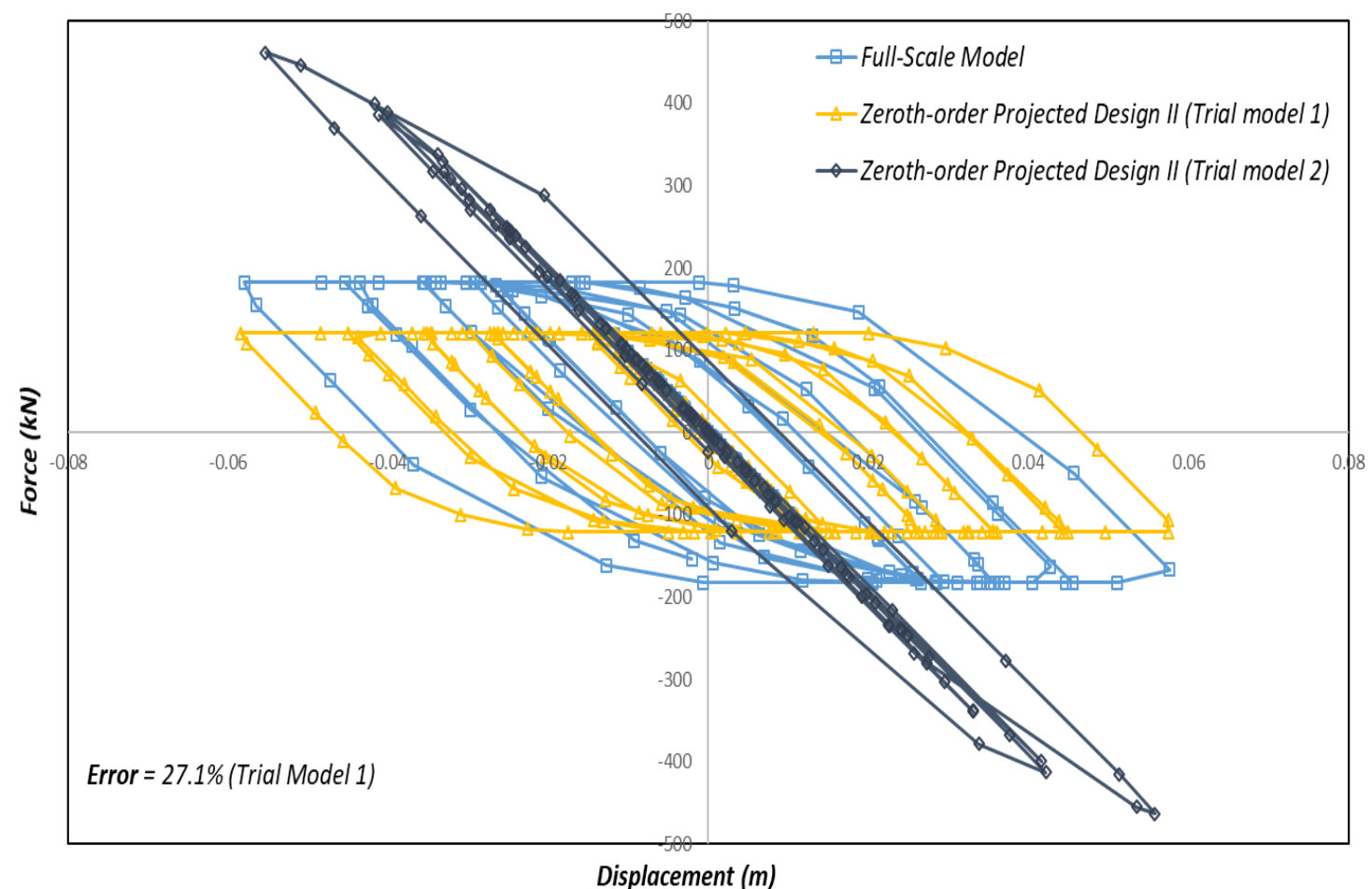

Figure 17. Zeroth order cyclic analysis of Design II models.

The percentage errors indicated in these figures refers to the differences in the areas enclosed by the last complete loop between the virtual and full-scale models. In view of the failure of the zeroth-order theory to provide a sufficiently close-enough match first-order theory is applied to both Designs I and II and the results presented in Fig. 18. A marked improvement is revealed in this figure with both designs providing an improved level of accuracy. This 
result provides good evidence for the benefits of two scaled experiments over one and confirms that the finite similitude theory is able to interpret the information arising from the two experiments.

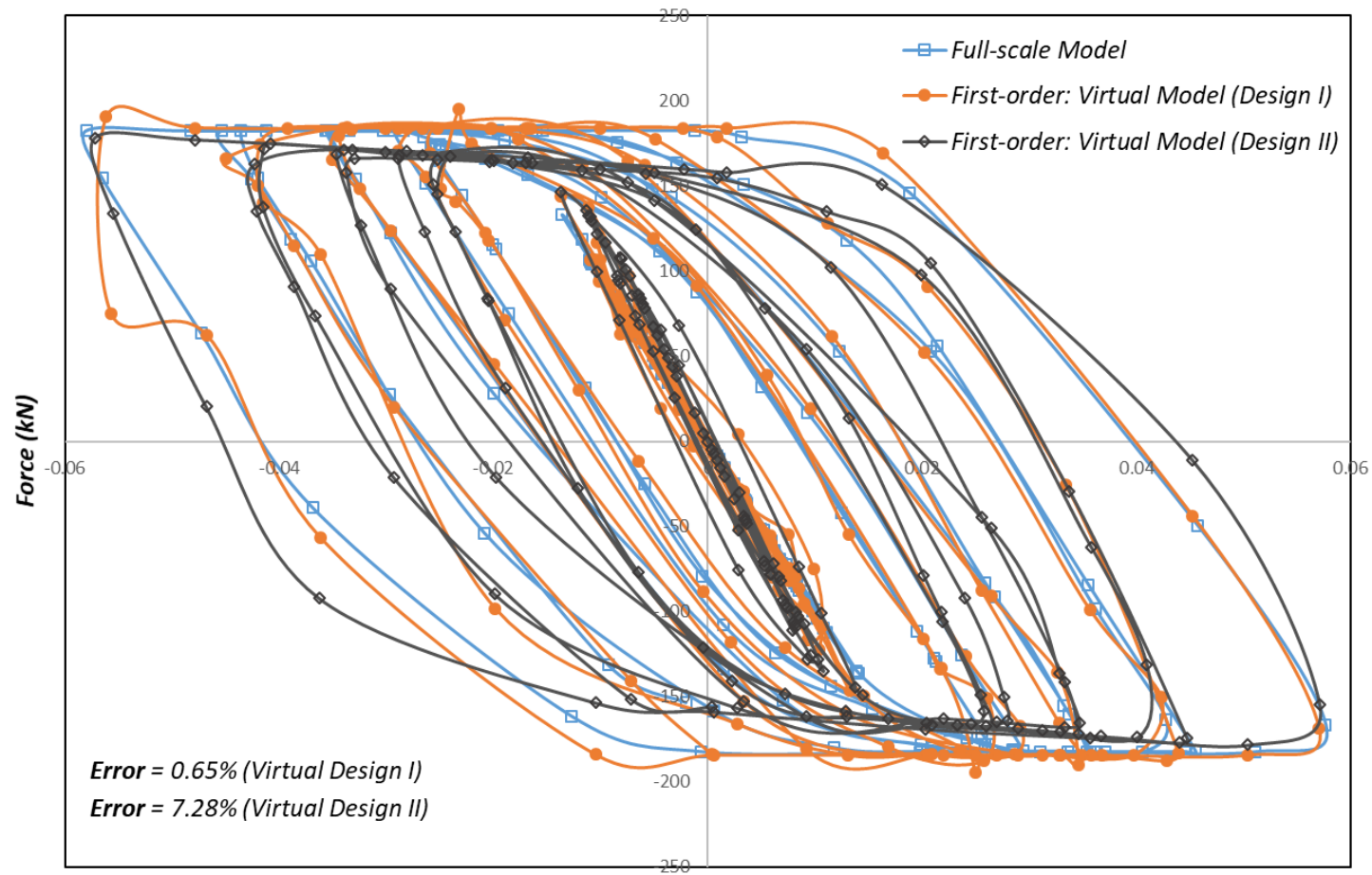

Displacement ( $m$ )

Figure 18. First-order cyclic analysis of Design I and II models.

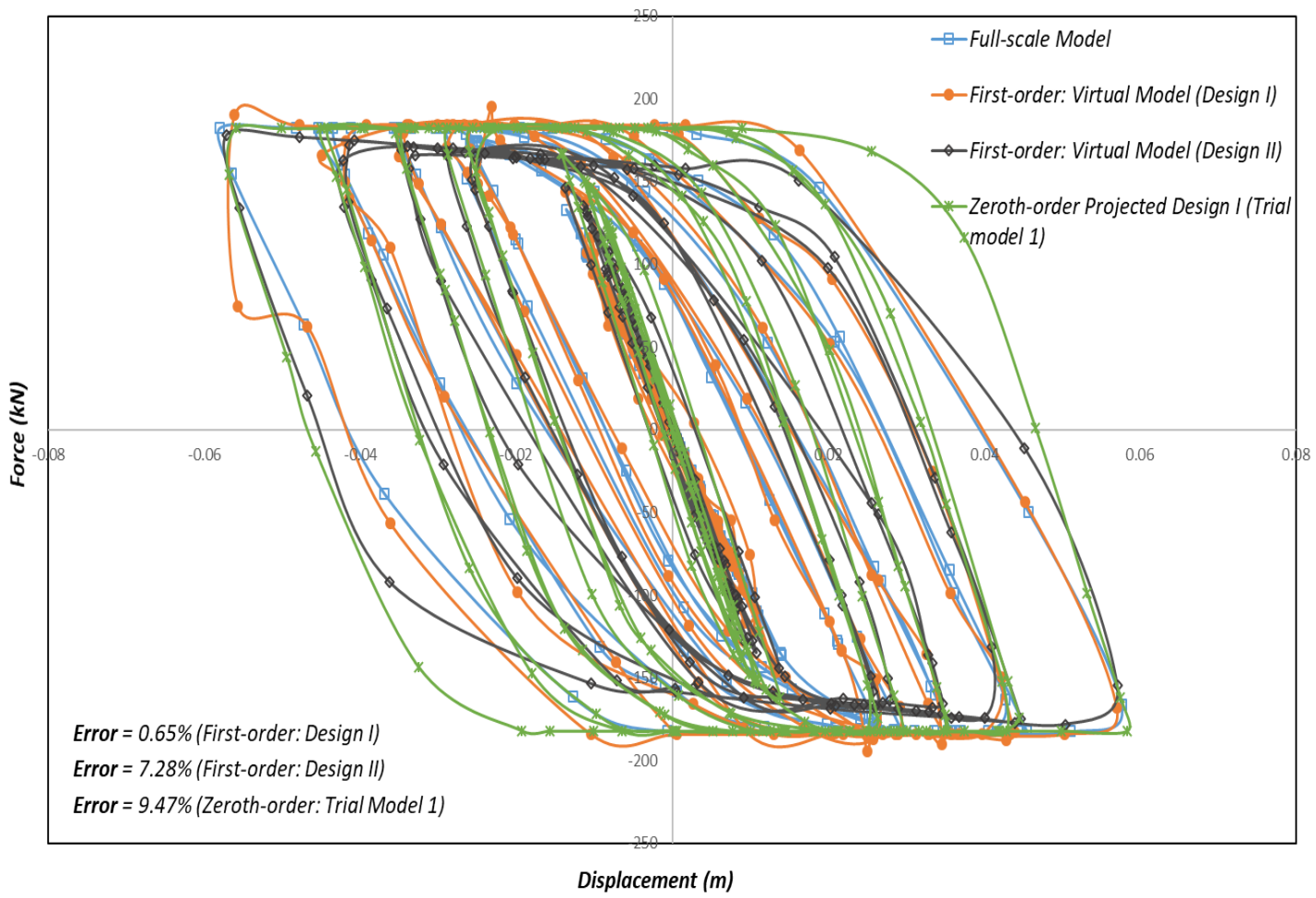

Figure 19. Zeroth and first-order cyclic analysis of Design I and models. 
A final comparison between the best zeroth-order and first-order finite similitude designs is provided in Fig. 19. The figure confirms that the first-order theory provides better predictions than zeroth order and the differences can be marked.

\section{CONCLUSION}

The paper is concerned with the assessment of a new scaling theory for the investigation of the aseismic behaviour of structures. The new approach involves two scaled experiments and is founded on the metaphysical concept of space scaling. The following conclusions can be drawn from the work presented in the paper:

1. A new scaling theory has been established that captures either explicitly or implicitly all scale dependencies that arise in structural mechanics.

2. A new form of similarity has been established (termed first-order finite similitude) in differential form (see Eq. 10), which can be integrated exactly using finite differences to combine results from two scaled-trial experiments to predict full-scale structural behaviour.

3. The new theory has been trialled on analytical and numerical models and provides predictions (sometimes markedly) superior to a single-scale trial experiment.

4. Scale effects as previously defined by dimensional analysis can up to a limited degree be accommodated (e.g. dimensionless strain need not be constant).

5. A new proportional theory has been established that has been shown to provide an efficient means to determine scaling parameters and avoid the need for expensive iterative procedures.

More specifically for the trial experiments performed it has been shown that:

1. The first-order scaling theory was able to target more material properties for matching between the virtual and physical full-scale model (e.g. yield stress, Young's modulus and density were matched in earthquake resistant structures).

2. The benefit of matching yield stress and Young's modulus for the cyclic loading of a two bay, three story structure was significant and confirmed the improvements possible with the new approach.

3. The investigation of aseismic structures is improved using two scaled experiments as opposed to a single scaled experiment. For a cyclically loaded three-storey building, the proposed methodology reduced an overall error of $9.47 \%$ with a single scaled experiment to $0.65 \%$ with the combination of two scaled experiments. 


\section{ACKNOWLEDGEMENTS}

The authors would like to acknowledge The Ministry of National Education in Turkey and the Department of Civil Engineering at Firat University for providing support for Muhammed Atar to facilitate his doctoral research at the University of Manchester. 


\section{REFERENCES}

[1] L. I. Sedov, M. Friedman, M. Holt, and J. D. Cole, "Similarity and Dimensional Methods in Mechanics," J. Appl. Mech., 1961.

[2] E. Buckingham, "On physically similar systems; Illustrations of the use of dimensional equations," Phys. Rev., vol. 4, no. 4, pp. 345-376, Oct. 1914.

[3] S. J. Kline and J. R. Radbill, "Similitude and Approximation Theory," J. Appl. Mech., vol. 33, no. 1, pp. 238-238, 1966.

[4] E. Buckingham, "The principle of similitude ," Nature, vol. 96, no. 2406. Nature Publishing Group, pp. 396-397, 1915.

[5] A. Casaburo, G. Petrone, F. Franco, and S. De Rosa, "A Review of Similitude Methods for Structural Engineering," Applied Mechanics Reviews, vol. 71, no. 3. American Society of Mechanical Engineers (ASME), 01-May-2019.

[6] A. J. Dutson and K. L. Wood, "Using rapid prototypes for functional evaluation of evolutionary product designs," Rapid Prototyp. J., vol. 11, no. 3, pp. 125-131, Jul. 2005.

[7] J. W. Wissmunn, "Dynamic Stability of Space Vehicles. Structural Dynamics Model Testing.,” KIRTLAND AFB, 1968.

[8] Z. Luo, Y. Wang, Y. Zhu, X. Zhao, and D. Wang, "The similitude design method of thin-walled annular plates and determination of structural size intervals," Proc. Inst. Mech. Eng. Part C J. Mech. Eng. Sci., vol. 230, no. 13, pp. 2158-2168, Aug. 2016.

[9] G. J. Simitses and J. Rezaeepazhand, "Structural similitude and scaling laws for laminated beam-plates," in American Society of Mechanical Engineers, Aerospace Division (Publication) AD, 1992.

[10] P. R. Budarapu, X. Zhuang, T. Rabczuk, and S. P. A. Bordas, "Multiscale modeling of material failure: Theory and computational methods," Adv. Appl. Mech., vol. 52, pp. 1-103, Jan. 2019.

[11] W. Q. Chen, “The renaissance of continuum mechanics,” Journal of Zhejiang University: Science A, vol. 15, no. 4. Zhejiang University, pp. 231-240, 01-Apr-2014.

[12] Y. Chen, S. Shabanov, and D. L. McDowell, "Concurrent atomistic-continuum modeling of crystalline materials," J. Appl. Phys., vol. 126, no. 10, p. 101101, Sep. 2019.

[13] A. Sharma, G. R. Reddy, and K. K. Vaze, "Shake table tests on a non-seismically detailed RC frame structure," Struct. Eng. Mech., vol. 41, no. 1, pp. 1-24, 2012.

[14] H. Guerrero Bobadilla, T. Ji, and J. A. Escobar, "Experimental studies of a steel frame model with and without buckling-restrained braces," Rev. Ing. Sísmica, no. 95, pp. 33$52,2016$.

[15] H. Guerrero, T. Ji, J. A. Escobar, and A. Teran-Gilmore, "Effects of BucklingRestrained Braces on reinforced concrete precast models subjected to shaking table excitation,” Eng. Struct., vol. 163, pp. 294-310, May 2018. 
[16] M. N. Nader and A. Astaneh-Asl, "Shaking Table Tests of Rigid, Semirigid, and Flexible Steel Frames," J. Struct. Eng., vol. 122, no. 6, pp. 589-596, Jun. 1996.

[17] S. E. Kim, D. H. Lee, and C. Ngo-Huu, "Shaking table tests of a two-story unbraced steel frame," J. Constr. Steel Res., vol. 63, no. 3, pp. 412-421, Mar. 2007.

[18] S. Petry and K. Beyer, "Scaling unreinforced masonry for reduced-scale seismic testing," Bull. Earthq. Eng., vol. 12, no. 6, pp. 2557-2581, Nov. 2014.

[19] Y. Zou and X. L. Lu, "Shaking table model test on Shanghai World Financial Center," World Inf. Earthq. Eng., 2007.

[20] M. Moghaddam, R. Darvizeh, K. Davey, and A. Darvizeh, "Scaling of the powder compaction process,” Int. J. Solids Struct., vol. 144-145, pp. 192-212, Jul. 2018.

[21] H. Sadeghi, K. Davey, R. Darvizeh, and A. Darvizeh, "A scaled framework for strain rate sensitive structures subjected to high rate impact loading," Int. J. Impact Eng., vol. 125, pp. 229-245, Mar. 2019.

[22] A. Al-Tamimi, R. Darvizeh, and K. Davey, "Experimental investigation into finite similitude for metal forming processes," J. Mater. Process. Technol., vol. 262, pp. 622-637, Dec. 2018.

[23] R. Ochoa-Cabrero, T. Alonso-Rasgado, and K. Davey, "Scaling in biomechanical experimentation: A finite similitude approach,” J. R. Soc. Interface, 2018.

[24] H. Sadeghi, K. Davey, R. Darvizeh, and A. Darvizeh, "Scaled models for failure under impact loading," Int. J. Impact Eng., vol. 129, pp. 36-56, Jul. 2019.

[25] K. Davey, R. Darvizeh, and A. Al-Tamimi, "Scaled metal forming experiments: A transport equation approach,” Int. J. Solids Struct., vol. 125, pp. 184-205, Oct. 2017.

[26] K. Davey and R. Darvizeh, "Neglected transport equations: extended RankineHugoniot conditions and J -integrals for fracture," Contin. Mech. Thermodyn., vol. 28, no. 5, pp. 1525-1552, Sep. 2016.

[27] K. Davey, H. Sadeghi, R. Darvizeh, A. Golbaf, and A. Darvizeh, "A Finite Similitude Approach to Scaled Impact Mechanics," Int. J. Impact Eng., vol. 148, 2021.

[28] J. S. Wu, Analytical and Numerical Methods for Vibration Analyses. 2015.

[29] “EN 1993-1-1: Eurocode 3: Design of steel structures - Part 1-1: General rules and rules for buildings."

[30] M. A. Meyers, Dynamic behavior of materials. Wiley, 1994.

[31] G. R. Johnson and W. H. Cook, "Fracture characteristics of three metals subjected to various strains, strain rates, temperatures and pressures," Eng. Fract. Mech., vol. 21, no. 1 , pp. 31-48, 1985.

[32] “PEER Ground Motion Database - PEER Center.” [Online]. Available: https://ngawest2.berkeley.edu/spectras/273010/searches/251041/edit?fbclid=IwAR1xu 9ySZ4HcvctPnKqU-rDzgeawvghlwaPRWOQIVCgwJPd7gp5ObcMveUA. [Accessed: 22-Mar-2020]. 
[33] N. S. Kim, J. H. Lee, and S. P. Chang, "Equivalent multi-phase similitude law for pseudodynamic test on small scale reinforced concrete models," Eng. Struct., vol. 31, no. 4, pp. 834-846, 2009.

[34] U. manual Abaqus, "Version 6.14," Dassault Systèmes Simulia Corp., Provid. RI, 2014.

[35] A. Anbuchezian and G. Baskar, "Behaviour of Cold-Formed Steel Beams Under Cyclic Load Reversal,” Int. J. Eng. Sci., pp. 22-30, 2013.

[36] K. V. Spiliopoulos and K. D. Panagiotou, "A numerical procedure for the shakedown analysis of structures under cyclic thermomechanical loading," Arch. Appl. Mech., vol. 85, no. 9-10, pp. 1499-1511, Sep. 2015.

[37] A. Kauffman and A. Memari, "Performance Evaluation of Different Masonry Infill Walls with Structural Fuse Elements Based on In-Plane Cyclic Load Testing," Buildings, vol. 4, no. 4, pp. 605-634, Sep. 2014.

[38] Y. Wu, X. Song, C. Ventura, and F. Lam, "Modeling Hysteretic Behavior of Lateral Load-Resisting Elements in Traditional Chinese Timber Structures," J. Struct. Eng. (United States), vol. 146, no. 5, pp. 1-14, 2020. 


\section{APPENDIX A: PROCEDURES FOR APPLICATION}

Presented in this section are the procedures followed in Sections 4, 5 and 6 in order to apply zeroth and first order finite theories. The zeroth-order theories follows the instruction set:

(i) Determine the geometrical and material properties and also boundary and initial (i.e. loading) conditions of the physical model;

(ii) Determine the dimensional scaling factor $\beta_{1}$ (thus, geometrical properties), material properties and boundary conditions for the trial model;

(iii) Determine which properties must be fixed;

(iv) Determine the independent density and time scaling factors (i.e. $\alpha_{01}^{\rho}$ and $g_{1}$ );

(v) Calculate the initial (i.e. loading) conditions for the trial model;

(vi) Conduct experimental tests on the trial model and;

(vii) Lift the trial model response to predict the physical model response.

A similar looking set of instruction apply for the first-order finite similitude theory and these are:

(i) Determine the geometrical and material properties and also boundary and initial (i.e. loading) conditions for the physical model;

(ii) Determine the dimensional scaling factors $\beta_{1}$ and $\beta_{2}$ (thus, geometrical properties), material properties and boundary conditions for the first and second trial models;

(iii) Determine the density scaling factors (i.e. $\alpha_{01}^{\rho}$ and $\alpha_{02}^{\rho}$ ) using the zeroth order finite similitude relations (i.e. $\rho_{p s}=\alpha_{01}^{\rho} \beta_{1}^{3} \rho_{t s 1}$ and $\rho_{p s}=\alpha_{02}^{\rho} \beta_{2}^{3} \rho_{t s 2}$ );

(iv) Determine which properties must be fixed;

(v) Determine the time scaling factors (i.e. $g_{1}$ and $g_{2}$ ) and also $R_{1}$ by restricting the intended properties and also initial (i.e. loading) conditions;

(vi) Calculate the initial (i.e. loading) conditions of the first and second trial models;

(vii) Conduct the experimental tests on the first and second trial models and;

(viii) Combine trial models to produce a virtual model to predict the physical model behaviour. 


\section{APPENDIX B: FIELD RESTRICTIONS}

In this section a general understanding is provided pertaining to the proportional-fields assumption of Section 5.2 and the various restrictions placed on the fields for zeroth and firstorder theories. The solution spaces on which each depends are $\mathfrak{I}_{z o}, \mathfrak{J}_{\text {lin }}, \mathfrak{I}_{f o}$ and $\mathfrak{I}_{d p}$, where $\mathfrak{I}_{z o}$ is zeroth-order solutions, $\mathfrak{I}_{\text {lin }}$ is proportional solutions (e.g. Eq. (26)), $\mathfrak{I}_{f o}$ is firstorder solutions (e.g. Eqs. (17)) and $\mathfrak{I}_{d p}$ is solutions with proportional differences (e.g. $\left.\sigma_{p s}-\sigma_{t s 1}=\hat{b}\left(\sigma_{t s 1}-\sigma_{t s 2}\right)\right)$.

It is assumed here that the elements of these spaces are drawn from vector spaces over the real field $\mathfrak{R}$. More specifically for non-zero $\lambda \in \mathfrak{R}, \boldsymbol{h}_{1} \in \mathfrak{J}_{z o} \Leftrightarrow \boldsymbol{h}_{p s}=\lambda \boldsymbol{h}_{1}$ with $\lambda$ restricted to scalars provided by zeroth-order theory (see Table 1.) For the space $\mathfrak{J}_{\text {lin }}$ however, $\boldsymbol{h}_{1} \in \mathfrak{I}_{z o} \Leftrightarrow \boldsymbol{h}_{p s}=\lambda \boldsymbol{h}_{1}$ for $\forall \lambda \neq 0 \in \mathfrak{R}$ and evidently $\mathfrak{I}_{z o} \subset \mathfrak{I}_{l i n}$. Similarly for the space $\mathfrak{I}_{f o}$, $\boldsymbol{h}_{1}, \boldsymbol{h}_{2} \in \mathfrak{J}_{f o} \Leftrightarrow \boldsymbol{h}_{p s}-\boldsymbol{h}_{1}=\lambda\left(\boldsymbol{h}_{1}-\boldsymbol{h}_{2}\right)$, and where $\boldsymbol{h}_{p s}, \boldsymbol{h}_{1}, \boldsymbol{h}_{2}$ and $\lambda$ are restricted to be those common fields and scalars provided by first-order theory (see Eq. (17)). Finally $\boldsymbol{h}_{1}, \boldsymbol{h}_{2} \in \mathfrak{J}_{d p} \Leftrightarrow \boldsymbol{h}_{p s}-\boldsymbol{h}_{1}=\lambda\left(\boldsymbol{h}_{1}-\boldsymbol{h}_{2}\right), \forall \lambda \neq 0 \in \Re$ and any common field types $\boldsymbol{h}_{p s}, \boldsymbol{h}_{1}$ and $\boldsymbol{h}_{2}$ ; evidently $\mathfrak{I}_{f o} \subset \mathfrak{I}_{d p}$.

Proposition B.1 The solution spaces satisfy $\mathfrak{I}_{z o} \subset \mathfrak{J}_{\text {lin }} \subset \mathfrak{J}_{d p}$ and $\mathfrak{I}_{z o} \subset \mathfrak{I}_{f o} \subset \mathfrak{J}_{d p}$.

Proof B.1 $\mathfrak{J}_{z o}$ is a proper subset of $\mathfrak{J}_{l i n}$ since for example $\boldsymbol{u}_{p s}=\beta_{1}^{-1} \boldsymbol{u}_{t s 1}$ and $\boldsymbol{u}_{p s}=-\boldsymbol{u}_{t s 1}$ are in $\mathfrak{I}_{\text {lin }}$ but only $\boldsymbol{u}_{p s}=\beta_{1}^{-1} \boldsymbol{u}_{t s 1}$ is in $\mathfrak{I}_{z o} . \mathfrak{I}_{\text {lin }}$ is a subset of $\mathfrak{I}_{d p}$ since $\boldsymbol{\sigma}_{t s 1}=\hat{b}_{1} \boldsymbol{\sigma}_{p s}$ and $\sigma_{t s 2}=\hat{b}_{2} \sigma_{p s}$ provide $\sigma_{p s}-\sigma_{t s 1}=\left(1-\hat{b}_{1}\right) \sigma_{p s}$ and $\sigma_{t s 1}-\sigma_{t s 2}=\left(\hat{b}_{1}-\hat{b}_{2}\right) \sigma_{p s}$. It is a proper subset since for any arbitrary non-zero $\boldsymbol{d}_{p s}$ not proportional to $\boldsymbol{\sigma}_{p s}$ the relationships $\sigma_{t s 1}=\sigma_{p s}-\hat{b} d_{p s} \quad$ and $\quad \sigma_{t s 2}=\left(1-\hat{b}^{-1}\right) \sigma_{p s}+(1-\hat{b}) d_{p s} \quad$ are not in $\mathfrak{J}_{l i n}$ but satisfy $\sigma_{p s}-\sigma_{t s 1}=\hat{b}\left(\sigma_{t s 1}-\sigma_{t s 2}\right)$, so belongs to $\mathfrak{I}_{d p}$. In addition $\mathfrak{I}_{f o}$ is a proper subset of $\mathfrak{I}_{d p}$ since for example $\quad \boldsymbol{u}_{p s}=\beta_{1}^{-1} \boldsymbol{u}_{t s 1}+R_{1}\left(\beta_{1}^{-1} \boldsymbol{u}_{t s 1}-\beta_{2}^{-1} \boldsymbol{u}_{t s 2}\right) \quad$ belongs to $\mathfrak{J}_{f o}$ but $\boldsymbol{u}_{p s}=-\boldsymbol{u}_{t s 1}+R_{1}\left(\beta_{1}^{-1} \boldsymbol{u}_{t s 1}-\beta_{2}^{-1} \boldsymbol{u}_{t s 2}\right)$ does not.

A question remains about the relationship between $\mathfrak{I}_{\text {lin }}$ and $\mathfrak{I}_{f o}$ but solutions of $\mathfrak{I}_{\text {lin }}$ can belong to $\mathfrak{I}_{f o}$ since $\mathfrak{I}_{z o} \subset \mathfrak{I}_{\text {lin }}$ but also there exists solutions that do not. An example is 
$\boldsymbol{h}_{p s}=-\boldsymbol{h}_{t s 1}$ and $\boldsymbol{h}_{p s}=-\boldsymbol{h}_{t s 2}$ since $\boldsymbol{h}_{p s}-\boldsymbol{h}_{t s 1}=2 \boldsymbol{h}_{p s}$ and $R_{1}\left(\boldsymbol{h}_{t s 1}-\boldsymbol{h}_{t s 2}\right)=\mathbf{0}$, so does no belong to $\mathfrak{\Im}_{f o}$ since $R_{1} \neq 0$. 